Алгебра и анализ

Том 20 (2008), № 3
St. Petersburg Math. J.

Vol. 20 (2009), No. 3, Pages 381-405

S 1061-0022(09)01053-X

Article electronically published on April 7, 2009

\title{
VOLUMES AND AREAS OF LIPSCHITZ METRICS
}

\author{
S. V. IVANOV
}

\begin{abstract}
Methods of estimating (Riemannian and Finsler) filling volumes by using nonexpanding maps to Banach spaces of $L^{\infty}$-type are developed and generalized. For every Finsler volume functional (such as the Busemann volume or the HolmesThompson volume), a natural extension is constructed from the class of Finsler metrics to all Lipschitz metrics, and the notion of area is defined for Lipschitz surfaces in a Banach space. A correspondence is established between minimal fillings and minimal surfaces in $L^{\infty}$-type spaces. A Finsler volume functional for which the Riemannian and the Finsler filling volumes are equal is introduced; it is proved that this functional is semielliptic.
\end{abstract}

\section{INTRODUCTION}

0.1. Motivations. This paper is motivated by filling minimality problems and boundary rigidity problems for Riemannian manifolds. Let $(M, g)$ be a compact Riemannian manifold with boundary $S=\partial M$. We denote by $d_{g}$ the associated distance function, $d_{g}: M \times M \rightarrow \mathbb{R}$.

Let $d: S \times S \rightarrow \mathbb{R}$ be an arbitrary metric on $S$. We say that a compact Riemannian manifold $(M, g)$ is a filling of a metric space $(S, d)$ if $d_{g}(x, y) \geq d(x, y)$ for all $x, y \in S$. The filling volume $\operatorname{FillVol}(S, d)$ of $(S, d)$ is defined by

$$
\operatorname{FillVol}(S, d)=\inf \{\operatorname{vol}(M, g):(M, g) \text { is a filling of }(S, d)\} \text {; }
$$

cf. [17. (This definition makes sense only for null-cobordant manifolds $S$; in general, $M$ should range over all pseudomanifolds or complete noncompact manifolds.) We say that $(M, g)$ is a minimal filling if it realizes the above infimum, that is, $\operatorname{vol}(M, g)=$ $\operatorname{FillVol}\left(\partial M,\left.d_{g}\right|_{S \times S}\right)$.

Many classical inequalities can be formulated in terms of minimal fillings. For instance, Besicovitch's inequality [5] means that a bounded region in $\mathbb{R}^{n}$ with the Euclidean metric is a minimal filling of its boundary (equipped with either the Euclidean or the $\ell_{\infty}$ metric), and Pu's inequality 22 is equivalent to the fact that the standard hemisphere is a minimal filling of an intrinsic metric of the circle (within the class of fillings homeomorphic to a disk).

Definition 0.1. Let $(M, g)$ be a Riemannian manifold (possibly with boundary). We say that $(M, g)$ has the geodesic minimality property if every geodesic segment is a shortest curve among all curves with the same endpoints.

We say that $(M, g)$ has the strong geodesic minimality property if the geodesic minimality property is satisfied for some manifold containing $M$ in the interior (and equipped with an extension of the metric $g$ ).

2000 Mathematics Subject Classification. Primary 53B40.

Key words and phrases. Filling volume, Finsler volume functional, (strong) geodesic minimality property.

Supported by RFBR (grant no. 05-01-00939). 
Recent results (see [19, 10]) indicate that the following conjecture is plausible.

Conjecture 0.2. If a manifold $(M, g)$ has the geodesic minimality property, then it is a minimal filling. Moreover, if $(M, g)$ has the strong geodesic minimality property, then it is a unique (up to isometry) minimal filling of its boundary.

Substitution of the definition of a minimal filling yields the following formulation of the conjecture. Suppose that $(M, g)$ has the strong geodesic minimality property and let a Riemannian manifold $\left(M^{\prime}, g^{\prime}\right)$ be such that $\partial M^{\prime}=\partial M=S$ and $\left.d_{g^{\prime}}\right|_{S \times S} \geq\left. d_{g}\right|_{S \times S}$. Then $\operatorname{vol}\left(M^{\prime}, g^{\prime}\right) \geq \operatorname{vol}(M, g)$ and in the case of equality the manifolds $(M, g)$ and $\left(M^{\prime}, g^{\prime}\right)$ are isometric.

It is easy to check that, if Conjecture 0.2 is true for $(M, g)$, then $g$ is uniquely determined (up to isometry) by the boundary distance function $\left.d_{g}\right|_{S \times S}$. Thus, Conjecture 0.2 would imply the following well-known Michel's boundary rigidity conjecture [21.

Conjecture 0.3. If $(M, g)$ has the strong geodesic minimality property, and if a manifold $\left(M^{\prime}, g^{\prime}\right)$ is such that $\partial M^{\prime}=\partial M=S$ and $\left.d_{g^{\prime}}\right|_{S \times S}=\left.d_{g}\right|_{S \times S}$, then $(M, g)$ and $\left(M^{\prime}, g^{\prime}\right)$ are isometric.

0.2. Auxiliary embeddings. M. Gromov [17 introduced a technique where filling volumes are estimated by using Kuratowski's construction, which makes it possible to embed any metric space $X$ isometrically in a Banach space $C^{0}(X) \subset \ell_{\infty}(X)$. (Applied to the problems we consider, this construction is described below in \$1.2) Variants of this construction were used in [19] and [10] to prove partial cases of Conjectures 0.2 and 0.3 In [17, Gromov showed that the filling volume of a Riemannian manifold $S$ equals, up to a multiplicative constant (depending on the dimension), the infimum of the areas of the surfaces spanning an isometric image of $S$ in a suitable Banach space. One of our purposes in this paper is to sharpen this result (in particular, to get rid of the constant), namely, to prove the following theorem.

Theorem 0.4. 1. Let d be a Lipschitz metric on a manifold $S$ (see Definition 2.1), and let $f$ be an isometric embedding of $(S, d)$ into the Banach space $\mathcal{L}=L^{\infty}(\mu)$, where $\mu$ is an arbitrary finite measure. Then, for a suitable definition of the notion of area in $\mathcal{L}$, the filling volume $\operatorname{FillVol}(S, d)$ equals the infimum of the areas of the Lipschitz surfaces spanning $f(S)$ in $\mathcal{L}$.

2. Let $(M, g)$ be a Riemannian manifold with boundary, and let $f:(M, g) \rightarrow \mathcal{L}$ be an isometric embedding $(\mathcal{L}$ is the same as above). Then $(M, g)$ is a minimal filling if and only if the surface $f(M)$ minimizes the area among all Lipschitz surfaces in $\mathcal{L}$ with the same boundary.

Remark 0.5 . For a manifold $M$ having the strong geodesic minimality property, there is a natural smooth map to $L^{\infty}(\partial M)$, namely, the boundary distance representation; see Example 1.9. It can be shown (as in [10]) that the surface defined by this map is minimal in the variational sense.

Remark 0.6. It is easy to formulate the equality case of Conjecture 0.2 in terms of auxiliary embeddings. Manifolds $(M, g)$ and $\left(M^{\prime}, g^{\prime}\right)$ with a common boundary are isometric if and only if their images under the boundary distance representation coincide.

The formulation of Theorem 0.4 is preliminary; the complete formulation is given in \$5 (Theorem 5.6 and Corollary [5.7). The definition of the area of a Lipschitz surface $f: M \rightarrow L^{\infty}(\mu)$ is a nontrivial issue, which is the subject of a major part of this paper. For such a definition to be useful, it should agree with both the extrinsic geometry of the surface (i.e., be computable in terms of derivatives of $f$ ) and with the intrinsic geometry (i.e., with the metric on $M$ induced by $f$ ). 
One of the difficulties to be met is the lack of Rademacher's theorem (about differentiability almost everywhere) for $L^{\infty}$-valued Lipschitz maps. Another difficulty is that the metrics induced on $M$ are not sufficiently regular (essentially, they are arbitrary Lipschitz metrics). In $\$ 2$ and $\$ 3$ we develop a technique for handling these difficulties. In 92 we consider the tangent Finsler structure of an arbitrary Lipschitz metric on $M$. With minor modifications, the construction goes along the same lines as in [15]; in $\$ 2$ our goal is to prove a technical results for further use. In $\$ 3$ we consider the notion of weak differentiability for a Lipschitz map $f: M \rightarrow L^{\infty}$ (this notion is obtained from the usual differentiability by switching to a weak topology) and prove a theorem about weak differentiability almost everywhere (Theorem 3.3). The main result of $\$ 3$ is that the weak differential of a map and the tangent Finsler structure of the induced metric on $M$ agree almost everywhere (Theorem 3.7).

0.3. Finsler volumes. Even for a smooth map of a manifold to a Banach space, the metric induced on the manifold is not Riemannian because the norms arising in tangent spaces are not Euclidean. Therefore, it is natural to consider filling volumes for Finsler metrics.

Recall that a (symmetric) Finsler structure on a smooth manifold $M$ is a continuous function $\Phi: T M \rightarrow \mathbb{R}_{+}$such that for every $p \in M$ the restriction $\left.\Phi\right|_{T_{p} M}$ is a norm. Finsler structures are also called Finsler metrics. A manifold equipped with a Finsler structure is called a Finsler manifold.

The Riemannian metrics are a special case of Finsler metrics. Namely, for a Riemannian manifold $M$, we can set $\Phi(v)$ to be equal to the Riemannian length of a tangent vector $v$. A Finsler metric $\Phi$ is Riemannian if and only if its restrictions to all fibers of the tangent bundle are Euclidean norms.

Unlike the Riemannian case, there are different (nonequivalent) definitions of the volume for Finsler manifolds, for instance, the Busemann volume [12], the HolmesThompson volume [18, Gromov's mass and comass [17, etc. Different applications need different definitions of volume. At the same time, many properties do not depend on the choice of a specific definition and hold for all "natural" notions of volume. Being "natural" includes a set of requirements given in Definition 4.1; the most important one is the monotonicity of volume with respect to the metric.

In 4 we give the necessary definitions and construct an extension of a Finsler volume functional from the class of Finsler metrics to all Lipschitz metrics. This allows us to define the area of a Lipschitz surface in a Banach space. In $\$ 5$ we prove that the filling volume within the class of all Lipschitz metrics coincides with that within the class of smooth strictly convex Finsler metrics (Theorem 5.2). Also, there we prove an analog of Theorem 0.4 for Finsler manifolds (Theorem 5.6). The Riemannian version (Corollary 5.7) is obtained from the Finsler one by using a special definition of volume, namely the inscribed Riemannian volume (see Example 4.4).

In 96 we give a brief survey of semiellipticity problems for Finsler volumes and prove Theorem 6.2 which asserts that the inscribed Riemannian volume has the compression property: for every Banach space $X$ and every $n$-dimensional linear subspace $V \subset X$ there exists an area-nonincreasing linear projection $P: X \rightarrow V$.

0.4. Notation and conventions. Throughout the paper we use the following notation: $\omega_{n}$ denotes the Lebesgue measure of a unit ball in $\mathbb{R}^{n}$;

$\mathbb{R}_{\infty}^{n}$ denotes the normed vector space $\left(\mathbb{R}^{n},\|\cdot\|_{\infty}\right)$, where the norm $\|\cdot\|_{\infty}$ is defined by $\left\|\left(x_{1}, \ldots, x_{n}\right)\right\|_{\infty}=\max _{1 \leq i \leq n}\left|x_{i}\right|$. The distance determined by this norm is denoted by $d_{\infty}$; 
$\mathcal{N}(V)$ and $\mathcal{N}_{0}(V)$, where $V$ is a finite-dimensional vector space, denote the set of all norms and all seminorms on $V$, respectively. These sets are regarded with the topology of pointwise convergence (which is the same as uniform convergence on compact sets).

All measures on manifolds are assumed to be Borel, and the term "measurable" refers to measurability with respect to the Borel $\sigma$-algebra.

\section{§1. MetriC SPACES}

This section contains preliminaries from metric geometry. A detailed exposition of most subjects considered here can be found in the book [11]. All statements presented in this section are well known; however, the most general formulations of some facts are hard to find in the literature and we supply them with proofs.

We use the terms "metric space" and "metric" in an extended sense; namely, we allow zero distances between different points.

Definition 1.1. A metric on a set $X$ is a function $d: X \times X \rightarrow[0,+\infty)$ satisfying the following conditions.

1. $d(x, x)=0$ for all $x \in X$.

2. Symmetry: $d(x, y)=d(y, x)$ for all $x, y \in X$.

3. Triangle inequality: $d(x, y)+d(y, z) \geq d(x, z)$ for all $x, y, z \in X$.

A metric space is a set equipped with a metric on it.

If the metric $d$ is clear from the context, we write $|x y|$ or $|x, y|$ instead of $d(x, y)$.

The standard definitions and theorems about metric spaces are easy to generalize to the case of metrics with zero distances. The metrics satisfying the condition $d(x, y)>0$ for $x \neq y$ (i.e., metrics in the usual sense) will be called positive metrics.

We often consider metrics or sequences of metrics defined on a set $X$ with a prescribed topology (for example, on a smooth manifold). In such a context, we always assume that a metric agrees with the topology in the following sense.

Definition 1.2. We say that a metric $d$ on a topological space $X$ agrees with the topology if the topology determined by $d$ is (nonstrictly) weaker than the topology of $X$.

It is easily seen that a metric $d$ agrees with the topology of $X$ if and only if the function $d: X \times X \rightarrow \mathbb{R}$ is continuous (with respect to the product topology).

\subsection{Isometric maps.}

Definition 1.3. Let $X, Y$ be metric spaces. A map $f: X \rightarrow Y$ is said to be nonexpanding if it does not increase distances, that is, $|f(x) f(y)| \leq|x y|$ for all $x, y \in X$.

A map $f: X \rightarrow Y$ is said to be isometric if it preserves the distances, that is, $|f(x) f(y)|=|x y|$ for all $x, y \in X$.

If $(Y, d)$ is a metric space and $X$ is an arbitrary set, then every map $f: X \rightarrow Y$ determines a metric $d^{\prime}$ on $X$ for which $f$ is isometric, namely, $d^{\prime}(x, y)=d(f(x), f(y))$ for all $x, y \in X$. We refer to $d^{\prime}$ as the metric induced by $f$ from $d$ and denote it by $f^{*} d$.

Note that the above notion of an isometric map differs from the terms "isometric embedding" and "isometric immersion" used in differential geometry, where one usually means preserving the lengths of curves and not the distances.

Kuratowski's well-known construction makes it possible to map any metric space $(X, d)$ isometrically to a Banach space. Namely, consider the space $C(X)$ of bounded continuous functions on $X$ with the standard norm $\|f\|=\sup _{X}|f|$ and fix a point $x_{0} \in X$. An isometric map $F: X \rightarrow C(X)$ is defined by $F(x)(y)=d(x, y)-d\left(x_{0}, y\right)$. In the case of a bounded $X$ one can use a simpler formula: $F(x)(y)=d(x, y)$. We need finite-dimensional approximations of this construction. 
Proposition 1.4. Let $(X, d)$ be a separable metric space. Then there exists a monotone nondecreasing sequence $\left\{d_{n}\right\}$ of metrics on $X$ converging to $d$ uniformly on compact sets and such that for every $n$, the space $\left(X, d_{n}\right)$ admits an isometric map to $\mathbb{R}_{\infty}^{n}$.

Proof. Let $P=\left\{p_{n}\right\}_{n=1}^{\infty}$ be a countable set dense in $X$. For each $n$, consider the function $f_{n}: X \rightarrow \mathbb{R}$ given by $f_{n}(x)=d\left(x, p_{n}\right)$ and define a map $F_{n}: X \rightarrow \mathbb{R}_{\infty}^{n}$ by

$$
F_{n}(x)=\left(f_{1}(x), f_{2}(x), \ldots, f_{n}(x)\right) .
$$

Define $d_{n}=F_{n}^{*} d_{\infty}$. Then $F_{n}$ is an isometric map of $\left(X, d_{n}\right)$ to $\mathbb{R}_{\infty}^{n}$. The construction shows that $d_{n+1}(x, y) \geq d_{n}(x, y)$, that is, $\left\{d_{n}\right\}$ is a monotone nondecreasing sequence.

It remains to prove that $d_{n}$ converges to $d$ uniformly on compact sets. The triangle inequality implies that the function $f_{n}$ is nonexpanding. Therefore,

$$
d_{n}(x, y)=\sup _{i \leq n}\left|f_{i}(x)-f_{i}(y)\right| \leq d(x, y) .
$$

Let $K$ be a compact subset of $X$, and let $\varepsilon>0$. Since $K$ is compact and $P$ is dense, there is an integer $n_{0}>0$ such that the set $\left\{p_{1}, p_{2}, \ldots, p_{n_{0}}\right\}$ is an $\varepsilon$-net for $K$. Let $x, y \in K$. Then there exists $i \leq n_{0}$ such that $d\left(x, p_{i}\right) \leq \varepsilon$. By the triangle inequality, $d\left(y, p_{i}\right) \geq d(x, y)-d\left(x, p_{i}\right) \geq d(x, y)-\varepsilon$. Hence,

$$
f_{i}(y)-f_{i}(x)=d\left(y, p_{i}\right)-d\left(x, p_{i}\right)>d(x, y)-2 \varepsilon,
$$

whence $d_{n}(x, y)=\left\|F_{n}(x)-F_{n}(y)\right\| \geq d(x, y)-2 \varepsilon$ for all $n \geq n_{0}$. Thus, $d-2 \varepsilon \leq d_{n} \leq d$ on $K$ for all $n \geq n_{0}$. Since $\varepsilon$ is arbitrary, this means that $d_{n}$ converges to $d$ uniformly on $K \times K$.

Remark 1.5. If the space $(X, d)$ is bounded, then the above maps $F_{n}: X \rightarrow \mathbb{R}_{\infty}^{n}$ converge in a natural sense to an isometric map $F: X \rightarrow \ell_{\infty}$. In the general case, these maps can be made convergent by subtracting a constant $d\left(x_{0}, p_{n}\right)$ from $f_{n}$, where $x_{0} \in X$ is a fixed point. Identifying $\ell_{\infty}$ with $\ell_{\infty}(P)$ and observing that the space $C(X)$ is isometrically mapped to $\ell_{\infty}(P)$ by the restriction operator, we easily see that the limit map $F$ coincides with Kuratowski's map.

\subsection{Extending Lipschitz maps.}

Proposition 1.6. Suppose $\mu$ is a measure on an arbitrary set $S, X$ is a separable metric space, $Y \subset X$, and $f: Y \rightarrow L^{\infty}(\mu)$ is a nonexpanding map. Then there exists a nonexpanding map $F: X \rightarrow L^{\infty}(\mu)$ such that $\left.F\right|_{Y}=f$.

Proof. We choose a countable dense subset $Y^{\prime} \subset Y$, and, for $x \in X$ and $s \in S$, put

$$
F(x)(s)=\inf \left\{f(y)(s)+|x y|: y \in Y^{\prime}\right\} .
$$

This formula defines a function $F(x): S \rightarrow[-\infty,+\infty]$ for every $x \in X$, and furthermore, this function is $\mu$-measurable, being the infimum of a countable collection of $\mu$-measurable functions. Observe that

$$
F(x)(s)=f(x)(s) \quad \text { for all } x \in Y^{\prime} \text { and } \mu \text {-almost all } s \in S .
$$

Indeed, for all $x, y \in Y^{\prime}$ and $\mu$-almost all $s \in S$ we have

$$
|f(x)(s)-f(y)(s)| \leq|x y|
$$

because $f$ is nonexpanding, whence $f(y)(s)+|x y| \geq f(x)(s)$. Taking the infimum over $y$ yields $F(x)(s) \geq f(x)(s)$. On the other hand,

$$
F(x)(s)=\inf \left\{f(y)(s)+|x y|: y \in Y^{\prime}\right\} \leq f(x)(s)+|x x|=f(x)(s)
$$

for every $x \in Y^{\prime}$, and (1.7) follows. 
Now we prove that

$$
\left|F(x)(s)-F\left(x^{\prime}\right)(s)\right| \leq\left|x x^{\prime}\right|
$$

for all $x, x^{\prime} \in X$ and $s \in S$ (if $F(x)(s)=F\left(x^{\prime}\right)(s)= \pm \infty$, we assume the difference to be zero). Indeed, for every $y \in Y^{\prime}$ we have

$$
\left|(f(y)(s)+|x y|)-\left(f(y)(s)+\left|x^{\prime} y\right|\right)\right|=|| x y|-| x^{\prime} y|| \leq\left|x x^{\prime}\right|
$$

by the triangle inequality. Therefore, the Hausdorff distance in $\mathbb{R}$ between the sets $T=\left\{f(y)(s)+|x y|: y \in Y^{\prime}\right\}$ and $T^{\prime}=\left\{f(y)(s)+\left|x^{\prime} y\right|: y \in Y^{\prime}\right\}$ does not exceed $|x y|$. Since $F(x)(s)=\inf T$ and $F\left(x^{\prime}\right)(s)=\inf T^{\prime}$, this implies (1.8).

Substituting an arbitrary point of $Y^{\prime}$ for $x^{\prime}$ in (1.8), we see that $F(x)(s)$ is finite for almost every $s \in S$. Thus $F$ is a map from $X$ to $L^{\infty}(\mu)$, and (1.8) means that this map is nonexpanding. It remains to observe that $f$ and $F$ agree on $Y$, because they agree on $Y^{\prime}$ by (1.7) and are continuous.

Example 1.9. Let $S$ be a closed smooth manifold, $d$ a metric on $S$, and $(M, g)$ a Riemannian manifold filling $(S, d)$, that is, $\partial M=S$ and $\left.d_{g}\right|_{S \times S} \geq d$ (see the Introduction). Consider the Kuratowski embedding $f:(S, d) \rightarrow L^{\infty}(S)$ given by $f(x)(y)=d(x, y)$. The inequality $\left.d_{g}\right|_{S \times S} \geq d$ implies that this map is nonexpanding with respect to the metric $d_{g}$. Therefore, there exists a nonexpanding map $F:(M, g) \rightarrow L^{\infty}(S)$ extending $f$.

Now suppose that $(M, g)$ has the geodesic minimality property. Then such a map $F:(M, g) \rightarrow L^{\infty}(S)$ can be defined by the formula $F(x)(s)=d_{g}(x, s), x \in M, s \in S$. It is easy to verify that this map is isometric. Indeed, for all $x, y \in M$ and $s \in S$ we have

$$
|F(x)(s)-F(y)(s)|=\left|d_{g}(x, s)-d_{g}(y, s)\right| \leq d_{g}(x, y)
$$

by the triangle inequality, whence $\|F(x)-F(y)\|_{\infty} \leq d_{g}(x, y)$. On the other hand, let $s_{0}$ be a point where the geodesic passing through $x$ and $y$ hits the boundary of the manifold. Then, by the geodesic minimality property, $d_{g}(x, y)=\left|d_{g}\left(x, s_{0}\right)-d_{g}\left(y, s_{0}\right)\right|$, i.e., $\left|F(x)\left(s_{0}\right)-F(y)\left(s_{0}\right)\right|=d_{g}(x, y)$, whence $\|F(x)-F(y)\|_{\infty}=d_{g}(x, y)$. The isometric map $F:(M, g) \rightarrow L^{\infty}(S)$ constructed in this way is referred to as the boundary distance representation of $(M, g)$.

Now, let $(M, g)$ and $\left(M^{\prime}, g^{\prime}\right)$ be as in Conjecture 0.2 that is, $(M, g)$ has the geodesic minimality property, $\partial M=\partial M^{\prime}=S$, and $\left.d_{g^{\prime}}\right|_{S \times S} \geq\left. d_{g}\right|_{S \times S}$. Then, as was shown above, there exists an isometric map $F:(M, g) \rightarrow L^{\infty}(S)$ and a nonexpanding map $F^{\prime}:\left(M^{\prime}, g^{\prime}\right) \rightarrow L^{\infty}(S)$ whose restrictions on $S$ coincide with Kuratowski's embedding of $\left(S,\left.d_{g}\right|_{S \times S}\right)$. For any natural definition of the area area $(F)$ of a surface $F: M \rightarrow L^{\infty}(S)$, the isometric maps should preserve the area and the nonexpanding maps should not increase it; hence area $(F)=\operatorname{vol}(M, g)$ and area $\left(F^{\prime}\right) \leq \operatorname{vol}\left(M^{\prime}, g^{\prime}\right)$. Therefore, to prove Conjecture 0.2 it suffices to verify the inequality area $(F) \leq$ area $\left(F^{\prime}\right)$. This argument proves one of the implications of Theorem 0.4 namely, that the area-minimality of a surface $F$ implies that $(M, g)$ is a minimal filling. The key requirement to the definition of area is the property that the nonexpanding maps do not increase area.

\subsection{Lengths of curves.}

Definition 1.10. A curve (or path) in a topological space $X$ is a continuous map $\gamma$ : $[a, b] \rightarrow X$, where $a \leq b$.

Note that if a metric $d$ on $X$ agrees with the topology (in the sense of Definition 1.2), then every curve in $X$ is continuous with respect to the metric $d$.

Definition 1.11. Let $(X, d)$ be a metric space, and $\gamma:[a, b] \rightarrow X$ a curve in $(X, d)$. A partition of $\gamma$ is a finite sequence of the form $\gamma\left(t_{0}\right), \ldots, \gamma\left(t_{n}\right)$, where $\left\{t_{i}\right\}_{i=0}^{n}$ is a partition of the segment $[a, b]$, that is, $a=t_{0} \leq t_{1} \leq \cdots \leq t_{n}=b$. The length of a partition 
$\gamma\left(t_{1}\right), \ldots, \gamma\left(t_{n}\right)$ is the sum $\sum_{i=0}^{n-1} d\left(\gamma\left(t_{i}\right), \gamma\left(t_{i+1}\right)\right)$. The length $L_{d}(\gamma)$ of $\gamma$ is the supremum of the lengths of all its partitions. We omit the index $d$ in the notation $L_{d}$ if the metric $d$ is clear from the context. A curve $\gamma$ is said to be rectifiable if $L(\gamma)<\infty$.

The standard properties of length (see, e.g., [11, §2.3]) can be generalized trivially to the case of metrics with zero distances. We shall need the following elementary facts.

Proposition 1.12. For every curve $\gamma:[a, b] \rightarrow X$ the following holds true.

1. Additivity: $L(\gamma)=L\left(\left.\gamma\right|_{[a, c]}\right)+L\left(\left.\gamma\right|_{[c, b]}\right)$ for all $c \in[a, b]$.

2. Triangle inequality: $L(\gamma) \geq|\gamma(a) \gamma(b)|$.

3. The length of a partition of $\gamma$ converges to $L(\gamma)$ as the mesh goes to zero. By the mesh of a partition $\left\{t_{i}\right\}$ of a segment $[a, b]$ we mean the number $\max _{i}\left|t_{i}-t_{i+1}\right|$.

Proposition 1.13. Let $\gamma$ be a curve in a metric space $(X, d)$, and let $\left\{d_{n}\right\}$ be a monotone nondecreasing sequence of metrics on $X$ converging to $d$ pointwise (that is, $d_{n}(x, y) \rightarrow$ $d(x, y)$ as $n \rightarrow \infty$ for all $x, y \in X)$. Then $L_{d_{n}}(\gamma) \rightarrow L_{d}(\gamma)$ as $n \rightarrow \infty$.

Proof. The inequality $d_{n} \leq d$ implies that $\gamma$ is continuous with respect to $d_{n}$ and $L_{d_{n}}(\gamma) \leq L_{d}(\gamma)$. It suffices to prove that $\liminf L_{d_{n}}(\gamma) \geq L_{d}(\gamma)$. Let $[a, b]$ be the domain of $\gamma$, and let $\left\{t_{i}\right\}_{i=0}^{N}$ be a partition of $[a, b]$. Summing up the inequalities $L_{d_{n}}\left(\left.\gamma\right|_{\left[t_{i}, t_{i+1}\right]}\right) \geq d_{n}\left(\gamma\left(t_{i}\right), \gamma\left(t_{i+1}\right)\right)$, we obtain the inequality

$$
L_{d_{n}}(\gamma) \geq \sum_{i=0}^{N-1} d_{n}\left(\gamma\left(t_{i}\right), \gamma\left(t_{i+1}\right)\right) .
$$

Passage to the limit as $n \rightarrow \infty$ yields

$$
\liminf L_{d_{n}}(\gamma) \geq \sum_{i=0}^{N-1} d\left(\gamma\left(t_{i}\right), \gamma\left(t_{i+1}\right)\right) .
$$

Taking the supremum over all partitions $\left\{t_{i}\right\}$ on the right, we see that $\liminf L_{d_{n}}(\gamma) \geq$ $L_{d}(\gamma)$, and the proposition follows.

Definition 1.14. A curve $\gamma:[a, b] \rightarrow X$ in a metric space is said to be absolutely continuous if for every $\varepsilon>0$ there exists $\delta>0$ such that for every finite collection $\left\{\left(a_{i}, b_{i}\right)\right\}_{i}$ of disjoint intervals contained in $[a, b]$ and satisfying $\sum_{i}\left|a_{i}-b_{i}\right| \leq \delta$, we have $\sum_{i}\left|\gamma\left(a_{i}\right) \gamma\left(b_{i}\right)\right|<\varepsilon$.

It is easy to check that every absolutely continuous curve is rectifiable. Obviously, the Lipschitz curves are absolutely continuous.

Definition 1.15. Let $\gamma:[a, b] \rightarrow X$ be a curve in a metric space, and let $t \in[a, b]$. Define the upper metric speed $\bar{s}_{\gamma}(t)$ of $\gamma$ at $t$ by

$$
\bar{s}_{\gamma}(t)=\limsup _{t^{\prime} \rightarrow t} \frac{\left|\gamma(t) \gamma\left(t^{\prime}\right)\right|}{\left|t-t^{\prime}\right|} .
$$

The similar lower limit is called the lower metric speed and is denoted by $\underline{s}_{\gamma}(t)$.

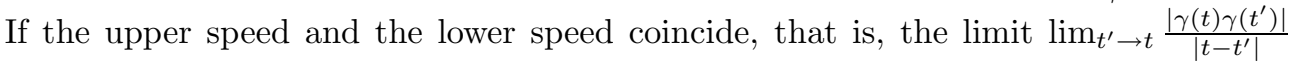
exists, then this limit is referred to as the metric speed of $\gamma$ at $t$ and is denoted by $s_{\gamma}(t)$.

Proposition 1.16. If a curve $\gamma:[a, b] \rightarrow X$ in a metric space $X$ is absolutely continuous, then the metric speed $s_{\gamma}(t)$ is well defined and finite for almost all $t \in[a, b]$, and

$$
L(\gamma)=\int_{a}^{b} s_{\gamma}(t) d t
$$


Proof. Define a (monotone nondecreasing) function $\lambda:[a, b] \rightarrow \mathbb{R}$ by $\lambda(t)=L\left(\left.\gamma\right|_{[a, t]}\right)$. Let $\varepsilon, \delta,\left\{\left(a_{i}, b_{i}\right)\right\}$ be as in Definition 1.14. Subdividing the intervals $\left(a_{i}, b_{i}\right)$ arbitrarily and substituting the resulting collections of segments into the same definition, we get the inequality $\sum_{i} L\left(\left.\gamma\right|_{\left[a_{i}, b_{i}\right]}\right) \leq \varepsilon$. Since $L\left(\left.\gamma\right|_{\left[a_{i}, b_{i}\right]}\right)=\lambda\left(b_{i}\right)-\lambda\left(a_{i}\right)$, it follows that the function $\lambda$ is absolutely continuous. Hence, it is differentiable almost everywhere on $[a, b]$, and

$$
L(\gamma)=\lambda(b)-\lambda(a)=\int_{a}^{b} \lambda^{\prime}(t) d t
$$

Thus, it suffices to prove that $\underline{s}_{\gamma}=\bar{s}_{\gamma}=\lambda^{\prime}$ almost everywhere on $[a, b]$. The inequality $L\left(\left.\gamma\right|_{\left[t, t^{\prime}\right]}\right) \geq\left|\gamma(t) \gamma\left(t^{\prime}\right)\right|$ implies that $\lambda^{\prime} \geq \bar{s}_{\gamma}$ wherever $\lambda^{\prime}$ is defined. So, it suffices to prove that $\underline{s}_{\gamma} \geq \lambda^{\prime}$ almost everywhere on $[a, b]$.

Suppose the contrary. Then there is an $\varepsilon>0$ and a Borel set $T \subset[a, b]$ of positive measure such that $\underline{s}_{\gamma}(t)<\lambda^{\prime}(t)-\varepsilon$ for all $t \in T$. By the regularity of the Lebesgue measure, $T$ contains a closed subset of positive measure, so that we may assume that $T$ itself is closed. We choose $\delta>0$ such that for every partition of $[a, b]$ of mesh at most $\delta$, the length of the corresponding partition of $\gamma$ differs from $L(\gamma)$ by a quantity less than $\frac{1}{3} \varepsilon \cdot m(T)$, where $m$ denotes the Lebesgue measure on $[a, b]$. For every $t \in T$, let $t_{1}=t_{1}(t) \in[a, b]$ be such that $\left|t_{1}-t\right|<\delta$ and

$$
\frac{\left|\gamma(t), \gamma\left(t_{1}\right)\right|}{\left|t-t_{1}\right|}<\frac{\left|\lambda(t)-\lambda\left(t_{1}\right)\right|}{\left|t-t_{1}\right|}-\varepsilon
$$

(such a $t_{1}$ exists because $\underline{s}_{\gamma}(t) \leq \lambda^{\prime}(t)-\varepsilon$ ). Then we extend the segment $\left[t, t_{1}\right]$ (or $\left[t_{1}, t\right]$ ) slightly, namely, include it in an open interval $(a(t), b(t))$, where $a(t)$ and $b(t)$ are so close to $t$ and $t_{1}$ that the above inequality holds true for $a(t)$ and $b(t)$, that is, $|a(t)-b(t)|<\delta$ and

$$
\frac{|\gamma(a(t)), \gamma(b(t))|}{|a(t)-b(t)|}<\frac{|\lambda(a(t))-\lambda(b(t))|}{|a(t)-b(t)|}-\varepsilon .
$$

The intervals of the form $(a(t), b(t))$ constitute an open covering of $T$; we choose a minimal finite subcovering $\left\{\left(a_{i}, b_{i}\right)\right\}_{i=1}^{N}$ of that covering. Then we change the enumeration of the intervals $\left(a_{i}, b_{i}\right)$ so that $a_{1} \leq a_{2} \leq \cdots \leq a_{N}$. The minimality of the covering implies that $\left(a_{i}, b_{i}\right) \cap\left(a_{j}, b_{j}\right)=\varnothing$ whenever $|i-j|>1$; in particular, the intervals indexed by even numbers are disjoint. We may assume that they cover at least half of the measure of $T$ (otherwise we use odd indices instead). By (1.17), we have

$$
\frac{\left|\gamma\left(a_{2 i}\right), \gamma\left(b_{2 i}\right)\right|}{\left|a_{2 i}-b_{2 i}\right|}<\frac{\left|\lambda\left(a_{2 i}\right)-\lambda\left(b_{2 i}\right)\right|}{\left|a_{2 i}-b_{2 i}\right|}-\varepsilon=\frac{L\left(\left.\gamma\right|_{\left[a_{2 i}, b_{2 i}\right]}\right)}{\left|a_{2 i}-b_{2 i}\right|}-\varepsilon,
$$

whence

$$
\sum_{i}\left(L\left(\left.\gamma\right|_{\left[a_{2 i}, b_{2 i}\right]}\right)-\left|\gamma\left(a_{2 i}\right), \gamma\left(b_{2 i}\right)\right|\right)>\varepsilon \cdot \sum_{i}\left|a_{2 i}-b_{2 i}\right| \geq \frac{1}{2} \varepsilon \cdot m(T) .
$$

Include the collection of segments $\left\{\left[a_{2 i}, b_{2 i}\right]\right\}$ in a partition $\left\{t_{j}\right\}$ of $[a, b]$ of mesh less than $\delta$. Then

$$
\begin{aligned}
L(\gamma) & -\sum_{j}\left|\gamma\left(t_{j}\right), \gamma\left(t_{j+1}\right)\right|=\sum_{j}\left(L\left(\left.\gamma\right|_{\left[t_{j}, t_{j+1}\right]}\right)-\left|\gamma\left(t_{j}\right), \gamma\left(t_{j+1}\right)\right|\right) \\
& \geq \sum_{i}\left(L\left(\left.\gamma\right|_{\left[a_{2 i}, b_{2 i}\right]}\right)-\left|\gamma\left(a_{2 i}\right), \gamma\left(b_{2 i}\right)\right|\right)>\frac{1}{2} \varepsilon \cdot m(T) .
\end{aligned}
$$

(Here the first inequality is obtained by removing the summands for which the segment $\left[t_{j}, t_{j+1}\right]$ is not one of the segments $\left[a_{2 i}, b_{2 i}\right]$.) But by the choice of $\delta$, the left-hand side does not exceed $\frac{1}{3} \varepsilon \cdot m(T)$. This contradiction completes the proof. 


\section{§2. LIPSCHITZ METRICS}

2.1. Weak Finsler structures. In this section we construct an analog of a tangent cone for an arbitrary Lipschitz metric on a manifold. The construction is similar to that in [15] but differs in some details.

In the sequel, $M$ denotes a smooth manifold (possibly with boundary) and $d_{\text {riem }}$ is an auxiliary Riemannian metric on $M$ chosen arbitrarily.

Definition 2.1. We say that a curve $\gamma:[a, b] \rightarrow M$ is Lipschitz if it is Lipschitz with respect to $d_{\text {riem }}$, that is, if there is a $C>0$ such that

$$
d_{\text {riem }}\left(\gamma(t), \gamma\left(t^{\prime}\right)\right) \leq C \cdot\left|t-t^{\prime}\right|
$$

for all $t, t^{\prime} \in[a, b]$.

We say that a metric $d$ on $M$ is Lipschitz if it is locally Lipschitz with respect to $d_{\text {riem }}$, that is, if for any point $x \in M$ there exists a neighborhood $U \ni x$ and $C>0$ such that

$$
d(y, z) \leq C \cdot d_{\text {riem }}(y, z)
$$

for all $y, z \in U$.

Clearly, this definition does not depend on the choice of the auxiliary Riemannian metric $d_{\text {riem. }}$. If $d$ is a Lipschitz metric and $\gamma$ is a Lipschitz curve, then $\gamma$ is Lipschitz (and therefore absolutely continuous) with respect to $d$. By Rademacher's theorem, every Lipschitz curve is differentiable almost everywhere on its domain.

Definition 2.2. A weak Finsler structure on a smooth manifold $M$ is a Borel function $\varphi: T M \rightarrow \mathbb{R}$ satisfying the following conditions.

1. Nonnegativity: $\varphi(v) \geq 0$ for all $v \in T M$.

2. Symmetry and positive homogeneity: $\varphi(\lambda v)=|\lambda| \varphi(v)$ for all $v \in T M, \lambda \in \mathbb{R}$.

3. Local boundedness: $\sup \left(\left.\varphi\right|_{K}\right)<\infty$ for any compact set $K \subset T M$.

Definition 2.3. Let $\varphi$ be a weak Finsler structure on $M$ and let $\gamma:[a, b] \rightarrow M$ be a Lipschitz curve. The length $L_{\varphi}(\gamma)$ of $\gamma$ with respect to $\varphi$ is defined by

$$
L_{\varphi}(\gamma)=\int_{a}^{b} \varphi\left(\gamma^{\prime}(t)\right) d t
$$

Definition 2.4. Let $d$ be a Lipschitz metric on $M$. For every $v \in T M$, we define a real number $\varphi_{d}(v)$ by

$$
\varphi_{d}(v)=\bar{s}_{\gamma}(0),
$$

where $\gamma$ is an arbitrary curve of the form $\gamma:(-\varepsilon, \varepsilon) \rightarrow M$ such that it is differentiable at zero and $\gamma^{\prime}(0)=v$. Here $\bar{s}$ denotes the upper metric speed with respect to $d$ (see Definition 1.15).

We refer to the function $\varphi_{d}: T M \rightarrow \mathbb{R}$ defined in this way as the tangent Finsler structure of the metric $d$.

The consistency of the above definition is ensured by the following lemma.

Lemma 2.5. Let $d$ be a Lipschitz metric on $M$, and let $\gamma_{1}$ and $\gamma_{2}$ be curves differentiable at zero and such that $\gamma_{1}^{\prime}(0)=\gamma_{2}^{\prime}(0)$. Then $\bar{s}_{\gamma_{1}}(0)=\bar{s}_{\gamma_{2}}(0)$ and $\underline{s}_{\gamma_{1}}(0)=\underline{s}_{\gamma_{2}}(0)$, where $\bar{s}$ and $\underline{s}$ denote the upper and the lower metric speeds with respect to $d$.

Proof. The definition of the metric speed and the triangle inequality imply that

$$
\left|\bar{s}_{\gamma_{1}}(0)-\bar{s}_{\gamma_{2}}(0)\right| \leq \limsup _{t \rightarrow 0} \frac{d\left(\gamma_{1}(t), \gamma_{2}(t)\right)}{|t|} .
$$

Since the metric $d$ is Lipschitz and $\gamma_{1}^{\prime}(0)=\gamma_{2}^{\prime}(0)$, for some constant $C$ we have

$$
d\left(\gamma_{1}(t), \gamma_{2}(t)\right) \leq C \cdot d_{\text {riem }}\left(\gamma_{1}(t), \gamma_{2}(t)\right)=o(|t|), \quad t \rightarrow 0 .
$$


Hence, the right-hand side of the previous inequality equals zero. The proof for the lower speed is similar.

Remark 2.6. Unlike a similar definition in [15, the function $\varphi_{d}$ as in Definition 2.4 is symmetric everywhere on $T M$, because the notion of the metric speed that we use (Definition 1.15) is symmetric with respect to the change of a parameter given by $t \mapsto-t$.

Proposition 2.7. Let $d$ be a Lipschitz metric on $M$, and $\varphi=\varphi_{d}$ its tangent Finsler structure. Then:

1. $\varphi$ is a weak Finsler structure in the sense of Definition 2.2.

2. For every $x \in M$, the restriction $\left.\varphi\right|_{T_{x} M}$ is Lipschitz.

3. For every Lipschitz curve $\gamma$, we have $L_{d}(\gamma)=L_{\varphi}(\gamma)$, where $L_{d}$ denotes the length with respect to the metric $d$ (see Definition 1.11) and $L_{\varphi}$ is the length with respect to $\varphi$ (see Definition 2.3).

4. Let $X$ be a normed vector space, and let $f:(M, d) \rightarrow X$ be an isometric map differentiable at a point $p \in M$. Then $\left.\varphi\right|_{T_{p} M}$ coincides with the seminorm induced from $\|\cdot\|_{X}$ by the map $d_{p} f$, that is,

$$
\varphi(v)=\left\|d_{p} f(v)\right\|_{X}
$$

for all $v \in T_{p} M$.

Proof. Assertions 1 and 2 follow trivially from the definitions and the fact that the metric is Lipschitz. Assertion 3 is a reformulation of Proposition 1.16,

We prove the fourth assertion. Let $\gamma:(-\varepsilon, \varepsilon) \rightarrow M$ be a curve differentiable at zero, $\gamma(0)=p, \gamma^{\prime}(0)=v$. Then

$$
\varphi(v)=\limsup _{t \rightarrow 0} \frac{d(\gamma(t), p)}{t}=\limsup _{t \rightarrow \infty} \frac{\|f(\gamma(t))-f(p)\|_{X}}{t}=\left\|(f \circ \gamma)^{\prime}(0)\right\|_{X}=\left\|d_{p} f(v)\right\|_{X} .
$$

Here the first identity follows from the definition of $\varphi$, the second from the fact that $f$ is isometric, and the last from the fact that $f$ is differentiable at $p$.

Definition 2.8. We say that two functions $\varphi_{1}, \varphi_{2}: T M \rightarrow \mathbb{R}$ coincide almost everywhere on Lipschitz curves if, for every Lipschitz curve $\gamma:[a, b] \rightarrow M$, the identity $\varphi_{1}\left(\gamma^{\prime}(t)\right)=$ $\varphi_{2}\left(\gamma^{\prime}(t)\right)$ is satisfied for almost all $t \in[a, b]$.

If two functions coincide almost everywhere on Lipschitz curves, then they obviously coincide almost everywhere on $T M$.

Proposition 2.9. Let $d$ be a Lipschitz metric on $M$, and let $\left\{d_{n}\right\}$ be a monotone nondecreasing sequence of metrics on $M$ converging to $d$ pointwise. Then

$$
\varphi_{d}=\lim _{n \rightarrow \infty} \varphi_{d_{n}}
$$

almost everywhere on Lipschitz curves.

Proof. Obviously, the tangent Finsler structure depends monotonically on the metric; hence, $\left\{\varphi_{d_{n}}\right\}$ is a monotone nondecreasing sequence bounded above by the function $\varphi_{d}$. Let $\gamma:[a, b] \rightarrow M$ be a Lipschitz curve. Proposition 1.13 implies that $L_{d_{n}}(\gamma) \rightarrow L_{d}(\gamma)$ as $n \rightarrow \infty$. Then Proposition 2.7(3) yields

$$
\int_{a}^{b} \varphi_{d}\left(\gamma^{\prime}(t)\right) d t=\lim _{n \rightarrow \infty} \int_{a}^{b} \varphi_{d_{n}}\left(\gamma^{\prime}(t)\right) d t=\int_{a}^{b} \lim _{n \rightarrow \infty} \varphi_{d_{n}}\left(\gamma^{\prime}(t)\right) d t
$$

(the second identity follows from Levy's theorem because the sequence $\left\{\varphi_{d_{n}}\right\}$ is monotone). Since $\lim _{n \rightarrow \infty} \varphi_{d_{n}} \leq \varphi_{d}$, it follows that the integrands are equal for almost all $t$.

The next proposition justifies the use of the term "Finsler structure" for $\varphi_{d}$. 
Proposition 2.10. Let $d$ be a Lipschitz metric on $M$, and let $\varphi=\varphi_{d}$ be its tangent Finsler structure. Then for almost all $x \in M$, the restriction $\left.\varphi\right|_{T_{x} M}$ is a seminorm.

Proof. First, we prove the statement in the case where the metric $d$ admits an isometric map to a finite-dimensional Banach space $X$. Let $f:(M, d) \rightarrow X$ be an isometric map. Then it is Lipschitz with respect to an auxiliary Riemannian metric on $M$, and therefore (by Rademacher's theorem), $f$ is differentiable almost everywhere on $M$. For every point $p \in M$ where $f$ is differentiable, Proposition 2.7(4) implies that $\varphi(v)=\left\|d_{x} f(v)\right\|_{X}$ for all $v \in T_{p} M$. Hence, $\left.\varphi\right|_{T_{p} M}$ is a seminorm.

In the general case, by Proposition 1.4 there exists a monotone nondecreasing sequence $\left\{d_{n}\right\}$ of metrics that admit isometric maps to finite-dimensional Banach spaces and converge to $d$. Applying the above proof to the metrics $d_{n}$, we see that the associated functions $\varphi_{n}=\varphi_{d_{n}}$ are seminorms on almost all fibers $T_{x} M, x \in M$. By Proposition 2.9. $\varphi=\lim _{n \rightarrow \infty} \varphi_{n}$ almost everywhere on $T M$. Thus, for almost all $x \in M$ the restriction $\left.\varphi\right|_{T_{x} M}$ coincides almost everywhere (on $T_{x} M$ ) with the limit of a sequence of seminorms. By continuity (see Proposition 2.7(2)), this means that $\left.\varphi\right|_{T_{x} M}$ itself is a seminorm.

Remark 2.11. In general, it is not true that $\left.\varphi\right|_{T_{x} M}$ is a seminorm for all $x \in M$. For example, it is easy to construct a Lipschitz metric $d$ on $\mathbb{R}^{2}$ such that $d((0,0),(0, x))=$ $d((0,0),(x, 0))=|x|$ and $d((0,0),(x, x))=10|x|$ for all $x \in \mathbb{R}$. Then, for the standard generators $e_{1}$ and $e_{2}$ of $T_{0} \mathbb{R}^{2}$, we have $\varphi_{d}\left(e_{1}\right)=\varphi_{d}\left(e_{2}\right)=1, \varphi_{d}\left(e_{1}+e_{2}\right) \geq 10$, so that the restriction of $\varphi_{d}$ to $T_{0} \mathbb{R}^{2}$ does not satisfy the triangle inequality.

The next proposition shows that the tangent Finsler structure is preserved essentially if a metric is replaced by an associated intrinsic one.

Proposition 2.12. Let $d$ be a Lipschitz metric on $M$. Let a metric $d^{*}$ on $M$ be defined by

$$
d^{*}(x, y)=\inf \left\{L_{d}(\gamma): \gamma \text { is a Lipschitz curve connecting } x \text { and } y\right\} .
$$

Then the tangent Finsler structures of $d$ and $d^{*}$ coincide almost everywhere on Lipschitz curves.

Proof. Denote $\varphi=\varphi_{d}, \varphi^{*}=\varphi_{d^{*}}$. Obviously, we have $d^{*} \geq d$, whence $\varphi^{*} \geq \varphi$. Let $\gamma:[a, b] \rightarrow M$ be a Lipschitz curve. Then $L_{d^{*}}(\gamma)=L_{d}(\gamma)$. Indeed, the inequality $d^{*} \geq d$ implies that $L_{d^{*}}(\gamma) \geq L_{d}(\gamma)$. To prove the reverse inequality, it suffices to check that for every partition $\left\{t_{i}\right\}$ of $[a, b]$ we have

$$
\sum d^{*}\left(\gamma\left(t_{i}\right), \gamma\left(t_{i+1}\right)\right) \leq L(\gamma)
$$

Observe that

$$
d^{*}\left(\gamma\left(t_{i}\right), \gamma\left(t_{i+1}\right)\right) \leq L_{d}\left(\left.\gamma\right|_{\left[t_{i}, t_{i+1}\right]}\right),
$$

because $\left.\gamma\right|_{\left[t_{i}, t_{i+1}\right]}$ belongs to the set of curves over which the infimum is taken in the definition of the distance $d^{*}$ on the left-hand side. Summing up such inequalities over all $i$, we obtain the desired one.

Thus, $L_{d^{*}}(\gamma)=L_{d}(\gamma)$, whence $L_{\varphi^{*}}(\gamma)=L_{\varphi}(\gamma)$ by Proposition 2.7(3). Since $\varphi^{*} \geq \varphi$, this implies that $\varphi^{*}\left(\gamma^{\prime}(t)\right)=\varphi(\gamma(t))$ for almost all $t \in[a, b]$.

Remark 2.13. The metric $d^{*}$ in Proposition 2.12 is a length metric, because it is determined by a length structure (see [11]). Another way to construct a length metric is the following: for $x, y \in M$, let $d^{\prime}(x, y)$ be equal to the infimum of the lengths of all continuous (not only Lipschitz) curves connecting $x$ and $y$. The tangent Finsler structure of such a metric $d^{\prime}$ also coincides with $\varphi_{d}$ almost everywhere on Lipschitz curves; this follows from the obvious inequalities $d \leq d^{\prime} \leq d^{*}$.

If the metric $d$ is bi-Lipschitz equivalent to a Riemannian one, then the metrics $d^{*}$ and $d^{\prime}$ coincide because every rectifiable curve admits a Lipschitz parametrization. 
2.2. Smoothing Lipschitz metrics. Let $(M, \varphi)$ be a Finsler manifold. The Finsler structure $\varphi$ determines a metric $d_{\varphi}$ on $M$ by $d_{\varphi}(x, y)=\inf \left\{L_{\varphi}(\gamma)\right\}$, where the infimum is taken over all Lipschitz (or piecewise smooth) curves $\gamma$ connecting the points $x$ and $y$ in $M$. A Finsler structure $\varphi$ and the associated metric $d_{\varphi}$ are said to be smooth if the function $\varphi: T M \rightarrow \mathbb{R}$ is smooth (that is, $C^{\infty}$ ) outside the zero section. A smooth Finsler structure $\varphi$ and the metric $d_{\varphi}$ are said to be strictly convex if for every point $x \in M$ the second differential of the function $\left.\varphi^{2}\right|_{T_{x} M}$ is positive definite everywhere on $T_{x} M \backslash\{0\}$.

Proposition 2.14. Let $M$ be a compact smooth manifold, $d$ a Lipschitz metric on $M$, $\varphi=\varphi_{d}$ its tangent Finsler structure. Then there exists a sequence $\left\{\varphi_{i}\right\}_{i=1}^{\infty}$ of smooth strictly convex Finsler structures on $M$ such that

1. $\left.\left.\varphi_{i}\right|_{T_{x} M} \rightarrow \varphi\right|_{T_{x} M}$ for almost all $x \in M$;

2. $d_{i}(x, y) \geq d(x, y)-\varepsilon_{i}$ for all $x, y \in M$, where $d_{i}=d_{\varphi_{i}}$, and $\left\{\varepsilon_{i}\right\}$ is a sequence of real numbers converging to zero;

3. $d_{i} \leq C \cdot d_{\text {riem }}$ for all $i$, where $d_{\text {riem }}$ is an auxiliary Riemannian metric and $C>1$ is a constant such that $d \leq(C-1) d_{\text {riem }}$.

Proof. Propositions 1.4 and 2.9 reduce the statement to the case where the metric $d$ admits an isometric map to $\mathbb{R}_{\infty}^{N}$. Such a map $f: M \rightarrow \mathbb{R}_{\infty}^{N}$ is Lipschitz with Lipschitz constant $C-1$ with respect to $d_{\text {riem }}$. Smoothing $f$ with a suitable convolution yields a sequence of smooth maps $f_{i}: M \rightarrow \mathbb{R}_{\infty}^{N}$ such that $f_{i} \rightrightarrows f, d f_{i} \rightarrow d f$ almost everywhere on $T M$, and $\left\|d f_{i}\right\| \leq C-\frac{1}{2}$, where the norm $\left\|d f_{i}\right\|$ is regarded with respect to the metric $d_{\text {riem }}$. We may assume that $N>2 \operatorname{dim} M$; then the maps $f_{i}$ can be replaced by smooth embeddings with the same properties. We approximate the norm $\|\cdot\|_{\infty}$ on $\mathbb{R}^{N}$ by smooth strictly convex norms $\|\cdot\|_{p_{i}}, p_{i} \rightarrow \infty$ (and all the $p_{i}$ are sufficiently large), where

$$
\left\|\left(x_{1}, \ldots, x_{N}\right)\right\|_{p}=\left(x_{1}^{p}+\cdots+x_{N}^{p}\right)^{1 / p} .
$$

Let $\varphi_{i}$ be a Finsler structure on $M$ induced by the map $f_{i}$ from the norm $\|\cdot\|_{p_{i}}$. Then $\left\{\varphi_{i}\right\}$ is a desired sequence. Indeed, the first requirement follows from the convergence $d f_{i} \rightarrow d f$ a.e. and the convergence $\|\cdot\|_{p} \rightarrow\|\cdot\|_{\infty}$ as $p \rightarrow \infty$. The second requirement follows from the relations

$$
d_{i}(x, y) \geq\left\|f_{i}(x)-f_{i}(y)\right\|_{p_{i}} \geq\left\|f_{i}(x)-f_{i}(y)\right\|_{\infty} \rightrightarrows\|f(x)-f(y)\|_{\infty}=d(x, y) .
$$

The third requirement follows from the condition $\left\|d f_{i}\right\| \leq C-\frac{1}{2}$ and the fact that the norms $\|\cdot\|_{p_{i}}$ and $\|\cdot\|_{\infty}$ are close to each other.

Remark 2.15. If the tangent Finsler structure $\varphi=\varphi_{d}$ in Proposition 2.14 is smooth and strictly convex in a neighborhood of a closed set $K \subset M$, then the approximating Finsler structures $\varphi_{i}$ can be chosen so that they coincide with $\varphi$ on $K$. To achieve this, it suffices to combine $\varphi$ and the structures $\varphi_{i}$ constructed in the proposition with the help of a smooth partition of unity.

\section{§3. WEAK DIFFERENTIABILITY}

3.1. Rademacher's theorem. Rademacher's classical theorem ([16, Theorem 3.1.6]) asserts that every Lipschitz map $f: \mathbb{R}^{m} \rightarrow \mathbb{R}^{n}$ is differentiable almost everywhere. This theorem is also true for functions with values in reflexive Banach spaces and, more generally, in Banach spaces having the Radon-Nikodým property (see [4, Chapter 5]).

However, for $L^{\infty}$-type spaces (which are used as target spaces for Kuratowski's embeddings) a similar assertion fails. For example, consider a map $f:[0,1] \rightarrow L^{\infty}[0,1]$ defined by $f(x)(y)=|x-y|$. It is easy to check that $f$ is Lipschitz with a Lipschitz constant 1 but nowhere differentiable. 
To avoid this difficulty, we introduce the notion of weak differentiability for maps with values in Banach spaces dual to separable ones. We are going to show that Lipschitz maps are weakly differentiable almost everywhere and that their weak differentials are related naturally to the tangent Finsler structures of induced metrics.

In the sequel, $M$ denotes a smooth manifold equipped with an auxiliary Riemannian metric $d_{\text {riem }}, X$ is a Banach space, and $X^{*}$ is the dual space (of continuous linear functionals $X \rightarrow \mathbb{R})$. In applications we usually set $X=L^{1}(\mu)$ and $X^{*}=L^{\infty}(\mu)$, where $\mu$ is a finite measure.

Definition 3.1. Let $f: M \rightarrow X^{*}$ be an arbitrary map. For every $u \in X$; consider the function $f_{u}: M \rightarrow \mathbb{R}$ given by

$$
f_{u}(x)=\langle f(x), u\rangle
$$

where $\langle$,$\rangle denotes the standard coupling of X^{*}$ and $X$. We say that $f$ is weakly differentiable at a point $p \in M$ if there exists a linear map $L: T_{p} M \rightarrow X^{*}$ such that for every $u \in X$ the function $f_{u}$ is differentiable at $p$ and its differential is given by the identity

$$
d_{p} f_{u}(v)=\langle L(v), u\rangle \text { for all } v \in T_{p} M .
$$

The map $L$ is referred to as the weak differential of $f$ at $p$ and is denoted by $d_{p}^{w} f$.

Proposition 3.2. Let $f: M \rightarrow X^{*}$ be a Lipschitz map (with respect to the metric $d_{\text {riem }}$ ), and let $p \in M$. Then the weak differentiability of $f$ at $p$ is equivalent to the property that for every $u \in X$ the function $f_{u}$ in Definition 3.1 is differentiable at $p$.

Proof. Let $C$ be a Lipschitz constant for $f$. Then $\|f(x)-f(y)\|_{X^{*}} \leq C \cdot d_{\text {riem }}(x, y)$ for every $x, y \in M$. Therefore,

$$
\left|f_{u}(x)-f_{u}(y)\right|=|\langle f(x)-f(y), u\rangle| \leq C \cdot\|u\|_{X} \cdot d_{\text {riem }}(x, y)
$$

for every $u \in X$, that is, the function $f_{u}$ is Lipschitz with a Lipschitz constant $C\|u\|_{X}$.

Suppose that $f_{u}$ is differentiable at $p$ for all $u \in X$. Fix a vector $v \in T_{p} M$. The Lipschitz continuity of $f_{u}$ implies the following derivative estimate: $d_{p} f_{u}(v) \leq C\|u\|_{X}|v|$. Observe that the function $u \mapsto d_{p} f_{u}(v)$ is linear and continuous, by the above estimate. Therefore, it represents an element $L(v)$ of $X^{*}$ such that

$$
d_{p} f_{u}(v)=\langle L(v), u\rangle \quad \text { for all } u \in X .
$$

Thus, we have constructed a map $L: T_{p} M \rightarrow X^{*}$. Obviously, it is linear and hence is the weak differential of $f$ at $p$.

Theorem 3.3. Let $X$ be a separable Banach space, and $f: M \rightarrow X^{*}$ a Lipschitz map. Then $f$ is weakly differentiable almost everywhere in $M$.

Proof. Since the assertion is local, we may assume that $M$ is a domain in $\mathbb{R}^{n}$ and $d_{\text {riem }}$ is the standard Euclidean metric. Let $U$ be a countable dense subset of $X$. For every $u \in U$ the function $f_{u}$ occurring in Definition 3.1 is Lipschitz and, hence, differentiable almost everywhere. Therefore, for almost every point $p \in M$ it is true that for all $u \in U$ the function $f_{u}$ is differentiable at $p$. We are going to show that for every such point $p$, the map $f$ is weakly differentiable at $p$. Let $C$ be a Lipschitz constant for $f$. Then for every $u \in X$ the function $f_{u}$ in Definition 3.1 is Lipschitz with constant $C\|u\|_{X}$. We fix a $u \in X$ and a sequence $\left\{u_{i}\right\} \subset U$ converging to $u$. By our assumption, every function $f_{u_{i}}$ is differentiable at $p$; we denote its differential $d_{p} f_{u_{i}}$ by $L_{i}\left(L_{i}: T_{p} M \rightarrow \mathbb{R}\right)$. For all $i$ and $j$, the function $f_{u_{i}}-f_{u_{j}}=f_{u_{i}-u_{j}}$ is Lipschitz with constant $C\left\|u_{i}-u_{j}\right\|_{X}$, whence $\left\|L_{i}-L_{j}\right\| \leq C\left\|u_{i}-u_{j}\right\| \rightarrow 0$ as $i, j \rightarrow \infty$. Therefore, the sequence $\left\{L_{i}\right\}$ converges to a linear function $L: T_{p} M \rightarrow M$. 
We choose $\varepsilon>0$ and fix $i$ such that $\left\|u-u_{i}\right\|_{X}<\varepsilon$; then $\left\|L-L_{i}\right\| \leq C \varepsilon$. Since $L_{i}=d_{p} f_{u_{i}}$, there is $\delta>0$ such that

$$
\left|f_{u_{i}}(q)-f_{u_{i}}(p)-L_{i}(q-p)\right|<\varepsilon|q-p|
$$

for all $q \in M \subset \mathbb{R}^{n}$ with $|q-p|<\delta$. By the Lipschitz continuity of $f$, we have $\|f(q)-f(p)\|_{X^{*}} \leq C \cdot|q-p|$, whence

$$
\begin{aligned}
& \left|\left(f_{u}(q)-f_{u}(p)\right)-\left(f_{u_{i}}(q)-f_{u_{i}}(q)\right)\right| \\
& \quad=\left|\left\langle f(q)-f(p), u-u_{i}\right\rangle\right| \leq C \cdot|q-p| \cdot|| u-u_{i} \|_{X} \leq C \varepsilon|q-p| .
\end{aligned}
$$

The inequality $\left\|L-L_{i}\right\| \leq C \varepsilon$ implies that

$$
\left|L(q-p)-L_{i}(q-p)\right| \leq C|q-p| .
$$

Adding (3.4), (3.5), and (3.6), we see that

$$
\left|f_{u}(q)-f_{u}(p)-L(q-p)\right|<(2 C+1) \varepsilon|q-p|
$$

whenever $|q-p|<\delta$. Since $\varepsilon$ is arbitrary, this means that the function $f_{u}$ is differentiable at $p$ and $d_{p} f_{u}=L$. Since $u$ is an arbitrary element of $X$, Proposition 3.2 implies that $f$ is weakly differentiable at $p$, hence the result.

3.2. Weak differential and metric. As in the preceding subsection, consider a Lipschitz map $f: M \rightarrow X^{*}$, where $M$ is a smooth manifold and $X$ is a separable Banach space. This map induces a metric $d$ on $M$ such that the map is isometric with respect to it (that is, $d(x, y)=\|f(x)-f(y)\|_{X^{*}}$ ).

If $f$ is differentiable at $p$ in the usual sense, then its differential induces a seminorm $\|\cdot\|_{p}$ on $T_{p} M,\|v\|_{p}=\left\|d_{p} f(v)\right\|_{X^{*}}$. By Proposition 2.7, this seminorm coincides with the tangent Finsler structure of $d$ at $p$.

In general, a similar identity for a weak differential fails. For example, consider $M=$ $[-1,1], X=L^{1}[0,1]$, and the map $f:[-1,1] \rightarrow X^{*}=L^{\infty}[0,1]$ given by

$$
f(x)(t)= \begin{cases}x \cdot \max \{1-t /|x|, 0\} & \text { if } x \neq 0, \\ 0 & \text { if } x=0 .\end{cases}
$$

It is easy to check that the map $f$ is isometric and weakly differentiable at zero, but $d_{0}^{w} f=0$.

Nevertheless, the following theorem shows that the tangent Finsler structure and the seminorm induced by the weak differential agree almost everywhere on $M$.

Theorem 3.7. Let $d$ be a Lipschitz metric on $M, \varphi=\varphi_{d}$ its tangent Finsler structure, $X$ a separable Banach space, and $f: M \rightarrow X^{*}$ an isometric map of the space $(M, d)$. Then for almost every point $p \in M$, we have

$$
\varphi_{d}(v)=\left\|d_{p}^{w} f(v)\right\|
$$

for all $v \in T_{p} M$.

Proof. Let $U=\left\{u_{i}\right\}_{i=1}^{\infty}$ be a countable set dense in the unit sphere of $X$. For every $\xi \in X^{*}$ we have

$$
\|\xi\|_{X^{*}}=\sup \left\{|\langle\xi, u\rangle|: u \in X,\|u\|_{X}=1\right\}=\sup _{i}\left|\left\langle\xi, u_{i}\right\rangle\right|,
$$

where the first identity follows from the definition of the norm $\|\cdot\|_{X^{*}}$ and the second from the fact that $\left\{u_{i}\right\}$ is dense in the unit sphere of $X$. 
For every positive integer $n$, consider the map $P_{n}: X^{*} \rightarrow \mathbb{R}_{\infty}^{n}$ given by $P_{n}(\xi)=$ $\left(\left\langle\xi, u_{1}\right\rangle,\left\langle\xi, u_{2}\right\rangle, \ldots,\left\langle\xi, u_{n}\right\rangle\right)$, and define a seminorm $\|\cdot\|_{n}$ on $X^{*}$ by $\|\xi\|_{n}=\left\|P_{n}(\xi)\right\|_{\infty}$. For every fixed $\xi \in X^{*}$ we have

$$
\|\xi\|_{n}=\max _{1 \leq i \leq n}\left|\left\langle\xi, u_{i}\right\rangle\right|
$$

therefore, the sequence $\left\{\|\xi\|_{n}\right\}_{n=1}^{\infty}$ is monotone nondecreasing and converges to $\|\xi\|_{X^{*}}$.

For every positive integer $n$ we define a Lipschitz metric $d_{n}$ on $M$ by $d_{n}(x, y)=$ $\|f(x)-f(y)\|_{n}$. Then $\left\{d_{n}\right\}$ is a monotone nondecreasing sequence of metrics converging to $d$ pointwise. If $\varphi_{n}$ denotes the tangent Finsler structure of $d_{n}$, then, by Proposition 2.9. $\left.\varphi_{n}\right|_{T_{p} M}$ converges to $\left.\varphi\right|_{T_{p} M}$ for almost all $p \in M$.

Let $p \in M$ be a point such that $\left.\varphi_{n}\right|_{T_{p} M}$ converges to $\left.\varphi\right|_{T_{p} M}$ and $f$ is weakly differentiable at $p$. Then for every $n$ the map $P_{n} \circ f: M \rightarrow \mathbb{R}_{\infty}^{n}$ is differentiable at $p$ and $d_{p}\left(P_{n} \circ f\right)=P_{n} \circ d_{p}^{w} f$, because the coordinate functions of the map $P_{n} \circ f$ are functions $f_{u_{i}}$ occurring in the definition of weak differentiability, $1 \leq i \leq n$. Observe that $P_{n} \circ f$ is an isometric map from $\left(M, d_{n}\right)$ to $\mathbb{R}_{\infty}^{n}$. Therefore, by Proposition $2.7(4),\left.\varphi_{n}\right|_{T_{p} M}$ equals the seminorm induced from the norm $\|\cdot\|_{\infty}$ by the map $d_{p}\left(P_{n} \circ f\right)$, or equivalently, the seminorm induced from the seminorm $\|\cdot\|_{n}$ on $X^{*}$ by the map $d_{p}^{w} f$. Hence, for every $v \in T_{p} M$ we have

$$
\varphi(v)=\lim _{n \rightarrow \infty} \varphi_{n}(v)=\lim _{n \rightarrow \infty}\left\|d_{p}^{w} f(v)\right\|_{n}=\left\|d_{p}^{w} f(v)\right\|_{X^{*}},
$$

and the theorem follows.

\section{$\S 4$. FinsLeR VOLUMeS}

4.1. Examples. Unlike the Riemannian case, there are different (nonequivalent) "natural" definitions of volume used for Finsler manifolds. By a "natural" definition we mean one for which the volume depends monotonically on the metric and agrees with the Riemannian volume on the class of Riemannian manifolds. Recall that a Finsler manifold is a smooth manifold with a continuous Finsler structure $\Phi: T M \rightarrow \mathbb{R}$ (unlike the weak Finsler structures defined in 42 , which are assumed measurable only).

Definition 4.1. Let $n$ be a fixed positive integer. We say that an $n$-dimensional Finsler volume functional is defined if, with every $n$-dimensional Finsler manifold $(M, \Phi)$, a Borel measure $\operatorname{vol}_{\Phi}$ on $M$ is associated so that the following conditions are satisfied.

1. The measure $\operatorname{vol}_{\Phi}$ depends monotonically on $\Phi$, that is, $\operatorname{vol}_{\Phi^{\prime}} \leq \operatorname{vol}_{\Phi}$ if $\Phi^{\prime} \leq \Phi$.

2. The measure is preserved by isometries, that is, if $(M, \Phi)$ and $\left(M^{\prime}, \Phi^{\prime}\right)$ are $n$ dimensional Finsler manifolds and $f: M \rightarrow M^{\prime}$ is an injective smooth map such that $\Phi=\Phi^{\prime} \circ d f$, then $\left.\operatorname{vol}_{\Phi^{\prime}}\right|_{f(M)}=f_{*} \operatorname{vol}_{\Phi}$, where the star denotes the push-forward of the measure by $f$.

3. If $M=\mathbb{R}^{n}$ and $\Phi$ is the standard Euclidean metric, then $\operatorname{vol}_{\Phi}$ is the standard Euclidean volume (i.e., the $n$-dimensional Lebesgue measure).

Example 4.2 (Busemann volume). The $n$-dimensional Hausdorff measure obviously satisfies the above requirements. In the Finslerian context, it is referred to as the Busemann volume. Busemann [12] proved that this is the only volume functional satisfying the following: the volume of a unit ball in an $n$-dimensional normed vector space equals $\omega_{n}$ (that is, does not depend on the norm). Despite being geometrically natural, this volume turns out to be inconvenient in many respects and does not possess some properties expected from the volume in differential and integral geometry (see, e.g., 2, 24]).

Example 4.3 (Holmes-Thompson volume). Let $(M, \Phi)$ be an $n$-dimensional Finsler manifold. Consider the cotangent bundle $T^{*} M$. In every fiber $T_{x}^{*} M$ there is a norm $\Phi_{x}^{*}$ 
dual to $\Phi_{x}$. The union of these norms is a continuous function $\Phi^{*}: T^{*} M \rightarrow \mathbb{R}$. Consider the set

$$
B^{*}(M, \Phi)=\left\{w \in T^{*} M: \Phi^{*}(w) \leq 1\right\}
$$

(i.e., the union of the unit balls of the norms $\Phi_{x}^{*}$ over all $x \in M$ ).

A canonical $2 n$-dimensional (symplectic) volume is defined on the cotangent bundle; we denote it by $V_{\text {symp. }}$. Now, we define a Finsler volume $\mathrm{vol}_{\Phi}^{s}$ by the formula

$$
\operatorname{vol}_{\Phi}^{s}(M)=\frac{1}{\omega_{n}} V_{\text {symp }}\left(B^{*}(M, \Phi)\right) .
$$

More precisely, the measure $\operatorname{vol}_{\Phi}^{s}$ is the push-forward of the measure $\frac{1}{\omega_{n}} V_{\text {symp }}$ by the projection map $B^{*}(M, \Phi) \rightarrow M$.

This volume functional is referred to as the Holmes-Thompson volume or the symplectic Finsler volume. It satisfies the requirements of Definition 4.1] because the construction is invariant and $\Phi^{*}$ depends on $\Phi$ antimonotonically.

This volume was introduced by Holmes and Thompson [18 in 1979, but problems related to it had been studied even before that. One of the reasons why it is convenient in differential geometry is the fact that the symplectic volume on the set $B^{*}(M, \Phi)$ corresponds to the Liouville measure on the unit tangent bundle and the latter is invariant under the geodesic flow.

Example 4.4 (inscribed Riemannian volume). Let $(M, \Phi)$ be a Finsler manifold. For every measurable $U \subset M$, define

$$
\operatorname{vol}_{\Phi}^{e}(U)=\inf \left\{\operatorname{vol}_{g}(U): g \text { is a Riemannian metric on } M, g \geq \Phi^{2}\right\},
$$

where $\operatorname{vol}_{g}$ denotes the Riemannian volume with respect to $g$. In the inequality $g \geq \Phi^{2}$, the Riemannian metric $g$ is regarded as a function on $T M$ that yields the square of the length of a tangent vector. We refer to the resulting measure $\operatorname{vol}_{\Phi}^{e}$ as the inscribed Riemannian volume of the Finsler metric $\Phi$. Obviously, this volume satisfies the requirements of Definition 4.1. Moreover, it is the maximal functional satisfying these requirements. This follows from the fact that a volume functional is defined uniquely within the class of Riemannian metrics (cf. Proposition 4.6).

There exists a unique Riemannian metric $g$ at which the infimum in (4.5) is attained. In every fiber $T_{x} M$, the unit ball of this metric $g$ is the maximal-volume ellipsoid contained in the unit ball of the norm $\Phi_{x}$ (the John ellipsoid [20]).

This volume is not convenient for the purposes of Finslerian geometry, but it turns out to be useful for Riemannian volume estimates that require auxiliary Finslerian constructions. It particular, it is this definition of volume that yields the coincidence of filling volumes within Riemannian metrics and within Finsler metrics, proved below in Theorem 5.2 .

It is easy to verify that the above three volume functionals are different. For example, consider the set $[-1,1]^{2}$ in the normed vector space $\mathbb{R}_{\infty}^{2}$. Its Busemann volume equals $\pi$ (since it is the unit ball of the norm), the Holmes-Thompson volume equals $\frac{8}{\pi}$, and the inscribed Riemannian volume equals 4.

Proposition 4.6. 1. An n-dimensional Finsler volume functional is uniquely determined by its values on the $n$-dimensional Banach spaces.

2. Any Finsler volume functional coincides with the Riemannian volume on the class of Riemannian manifolds.

Proof. The second requirement of Definition 4.1 allows us to limit ourselves to Finsler structures in $\mathbb{R}^{n}$. Let $\Phi$ be a Finsler structure in $\mathbb{R}^{n}$. Identifying all the tangent spaces $T_{x} \mathbb{R}^{n}$ with $\mathbb{R}^{n}$, we may regard $\Phi$ as a family of norms $\left\{\Phi_{x}\right\}_{x \in \mathbb{R}^{n}}$ in $\mathbb{R}^{n}$, where 
$\Phi_{x}=\left.\Phi\right|_{T_{x} \mathbb{R}^{n}}$. We fix $x_{0} \in \mathbb{R}^{n}$ and denote $\|\cdot\|=\Phi_{x_{0}}$. Let $\varepsilon>0$. Then, by the continuity of $\Phi$, there is a neighborhood $U$ of $x_{0}$ such that

$$
(1-\varepsilon)\|\cdot\| \leq \Phi_{x} \leq(1+\varepsilon)\|\cdot\|
$$

for all $x \in U$. For every $\lambda>0$ the space $\left(\mathbb{R}^{n}, \lambda\|\cdot\|\right)$ is isometric to $\left(\mathbb{R}^{n},\|\cdot\|\right)$ via a $\lambda$-homothety. Together with the second requirement of Definition 4.1, this implies that

$$
\operatorname{vol}_{\lambda\|\cdot\|}(A)=\operatorname{vol}_{\|\cdot\|}(\lambda A)
$$

for every measurable $A \subset \mathbb{R}^{n}$. The measure $\operatorname{vol}_{\|\cdot\|}$ is proportional to the Lebesgue measure because it is locally finite and translation invariant; hence, $\operatorname{vol}_{\|\cdot\|}(\lambda A)=\lambda^{n} \operatorname{vol}_{\|\cdot\|}(A)$. Thus, $\operatorname{vol}_{\lambda\|\cdot\|}=\lambda^{n} \operatorname{vol}_{\|\cdot\|}$. Substituting $\lambda=1 \pm \varepsilon$ and using the monotonicity of measure with respect to the metric, we see that

$$
(1-\varepsilon)^{n} \operatorname{vol}_{\|\cdot\|} \leq \operatorname{vol}_{\Phi} \leq(1+\varepsilon)^{n} \operatorname{vol}_{\|\cdot\|}
$$

within $U$. Sending $\varepsilon$ to zero shows that the density of $\operatorname{vol}_{\Phi}$ with respect to the Lebesgue measure at $x_{0}$ equals the density of $\operatorname{vol}_{\|\cdot\|}$. Thus the measure $\operatorname{vol}_{\Phi}$ has a density whose value at every point $x \in \mathbb{R}^{n}$ is determined by the volume functional on the Banach space $\left(\mathbb{R}^{n}, \Phi_{x}\right)$. The first assertion of the proposition follows.

If the Finsler structure $\Phi$ is actually Riemannian, then all the norms $\Phi_{x}, x \in \mathbb{R}^{n}$, are Euclidean. Since all $n$-dimensional Euclidean spaces are isometric, the second and third requirements of Definition 4.1 uniquely determine the density of $\operatorname{vol}_{\Phi}$, and it is equal to the density of the standard Riemannian volume. The second assertion of the proposition follows.

4.2. Finsler volume densities. Proposition 4.6 shows that, in order to define an $n$ dimensional Finsler volume functional, it suffices to define it on the $n$-dimensional Banach spaces. In this section we give the corresponding definitions following the approach of [3] and define a Finsler volume for arbitrary Lipschitz metrics.

Assume that we have fixed an $n$-dimensional Finsler volume functional $\Phi \mapsto \operatorname{vol}_{\Phi}$. In particular, for every $n$-dimensional normed vector space $(V,\|\cdot\|)$ we have an associated translation invariant and locally finite Borel measure vol $\|\cdot\|$ on $V$. All such measures on $V$ are proportional to one another. They are in a natural one-to-one correspondence with the norms on the $n$th exterior power $\Lambda^{n} V$; namely, the norm of an $n$-vector $v_{1} \wedge v_{2} \wedge \cdots \wedge v_{n}$ equals the measure of the parallelotope spanned by the vectors $v_{1}, v_{2}, \ldots, v_{n}$. We denote this norm of an $n$-vector $\sigma$ by $\operatorname{vol}_{\|\cdot\|}(\sigma)$.

Definition 4.1 implies that this map has the following properties.

(4.7) If $(V,\|\cdot\|)$ and $\left(V^{\prime},\|\cdot\|^{\prime}\right)$ are $n$-dimensional normed vector spaces and $f: V \rightarrow V^{\prime}$ is a nonexpanding linear map, then $\operatorname{vol}_{\|\cdot\|}(\sigma) \leq \operatorname{vol}_{\|\cdot\|^{\prime}}\left(f_{*}(\sigma)\right)$ for all $\sigma \in \Lambda^{n} V$, where the star denotes the natural action of an isomorphism on $n$-forms.

In particular, if $\|\cdot\|$ and $\|\cdot\|^{\prime}$ are norms on $V$ and $\|\cdot\| \leq\|\cdot\|^{\prime}$, then $\operatorname{vol}_{\|\cdot\|} \leq$ $\operatorname{vol}_{\|\cdot\|^{\prime}}$.

(4.8) If $|\cdot|$ is a Euclidean norm, then $\operatorname{vol}_{|\cdot|}$ is the corresponding Euclidean volume.

Definition 4.9. We say that an $n$-dimensional Banach volume functional is defined if with every $n$-dimensional normed vector space $(V,\|\cdot\|)$ a norm vol $\|\cdot\|$ on $\Lambda^{n} V$ is associated so that properties (4.7) and (4.8) are fulfilled.

Property (4.7) implies that the volume is preserved by isometries. Therefore, it suffices to define an $n$-dimensional Banach volume $\operatorname{vol}_{\|\cdot\|}$ only for the norms $\|\cdot\|$ on $\mathbb{R}^{n}$. For a fixed vector space $V$, the volume functional $\|\cdot\| \mapsto \operatorname{vol}_{\|\cdot\|}$ determines a map from $\mathcal{N}(V)$ to $\mathcal{N}\left(\Lambda^{n}(V)\right.$ ). (Recall that $\mathcal{N}(V)$ denotes the set of all norms on $V$. The space $\mathcal{N}\left(\Lambda^{n}(V)\right)$ is one-dimensional and can be identified with $\mathbb{R}_{+}$, but such an identification 
is not invariant.) This map can be extended naturally to the set $\mathcal{N}_{0}(V)$ of all seminorms; namely, we define $\operatorname{vol}_{\|\cdot\|}=0$ if the seminorm $\|\cdot\|$ is degenerate (i.e., is not a norm).

Lemma 4.10. Let $\|\cdot\| \mapsto \operatorname{vol}_{\|\cdot\|}$ be an $n$-dimensional Banach volume functional, and $V$ an $n$-dimensional vector space. Then:

1. $\operatorname{vol}_{\|\cdot\|}$ is a function of $\|\cdot\|$ homogeneous of degree $n$, that is, $\operatorname{vol}_{\lambda\|\cdot\|}=\lambda^{n} \operatorname{vol}_{\|\cdot\|}$ for every norm $\|\cdot\|$ on $V$ and every $\lambda>0$;

2. the map $\|\cdot\| \mapsto \operatorname{vol}_{\|\cdot\|}: \mathcal{N}_{0}(V) \rightarrow \mathcal{N}_{0}\left(\Lambda^{n} V\right)$ is continuous.

Proof. Statement 1 follows from the fact that the spaces $(V, \lambda\|\cdot\|)$ and $(V,\|\cdot\|)$ are isometric via a $\lambda$-homothety (see the proof of Proposition 4.6).

2. First, we prove continuity on the set $\mathcal{N}(V)$ of norms. Consider a sequence $\left\{\|\cdot\|_{i}\right\}_{i=1}^{\infty}$ of norms converging to a norm $\|\cdot\|$. The ratio $\frac{\|\cdot\|_{i}}{\|\cdot\|}$ converges uniformly to 1 ; that is,

$$
\left(1-\varepsilon_{i}\right)\|\cdot\| \leq\|\cdot\|_{i} \leq\left(1+\varepsilon_{i}\right)\|\cdot\|
$$

for some sequence $\varepsilon_{i} \rightarrow 0$. Hence, by homogeneity,

$$
\left(1-\varepsilon_{i}\right)^{n} \operatorname{vol}_{\|\cdot\|} \leq \operatorname{vol}_{\|\cdot\|_{i}} \leq\left(1+\varepsilon_{i}\right)^{n} \operatorname{vol}_{\|\cdot\|}
$$

so that $\operatorname{vol}_{\|\cdot\|_{i}} \rightarrow \operatorname{vol}_{\|\cdot\|}$, and continuity on $\mathcal{N}(V)$ follows.

Now, let $\|\cdot\|$ be a degenerate seminorm, and let $\left\{\|\cdot\|_{i}\right\}_{i=1}^{\infty}$ be a sequence of seminorms converging to $\|\cdot\|$. Let $V_{0}$ be the null subspace of $\|\cdot\|$, i.e., $V_{0}=\{v \in V:\|v\|=0\}$, and let $V_{1}$ be a subspace complementary to $V_{0}$. We may assume that $V_{0}=\mathbb{R}^{k}$ and $V_{1}=\mathbb{R}^{n-k}$, where $1 \leq k \leq n, V=\mathbb{R}^{k} \times \mathbb{R}^{n-k}=\mathbb{R}^{n}$. For every $\varepsilon>0$, consider the norm $\|\cdot\|_{(\varepsilon)}$ on $\mathbb{R}^{n}$ given by

$$
\|(x, y)\|_{(\varepsilon)}=\varepsilon|x|+2\|y\|, \quad x \in \mathbb{R}^{k}, y \in \mathbb{R}^{n-k},
$$

where $|\cdot|$ is the standard Euclidean norm. Since the restriction of $\|\cdot\|$ to $V_{1}$ is a norm, the ratio $\frac{\|\cdot\|_{i}}{\|\cdot\|}$ converges uniformly to 1 on $V_{1} \backslash\{0\}$, whence $\|\cdot\|_{i} \leq 2\|\cdot\|$ on $V_{1}$ for all sufficiently large $i$. We may assume that this inequality is satisfied for all $i$. Define

$$
\varepsilon_{i}=\max \left\{\|v\|_{i}: v \in V_{0},|v|=1\right\} .
$$

Then $\varepsilon_{i} \rightarrow 0$, because the restrictions of the seminorms $\|\cdot\|_{i}$ to $V_{0}$ converge to zero. The inequalities $\|\cdot\|_{i} \leq 2\|\cdot\|$ on $V_{1}$ and $\|\cdot\|_{i} \leq \varepsilon_{i}|\cdot|$ on $V_{0}$ imply that $\|\cdot\|_{i} \leq\|\cdot\|_{\left(\varepsilon_{i}\right)}$ everywhere on $V$.

Observe that all norms of the form $\|\cdot\|_{(\varepsilon)}$ are isometric; an isometry between $\|\cdot\|_{(1)}$ and $\|\cdot\|_{(\varepsilon)}$ is given by the map $(x, y) \mapsto(\varepsilon x, y), x \in \mathbb{R}^{k}, y \in \mathbb{R}^{n-k}$. Therefore, vol $\|_{\|\cdot\|_{(\varepsilon)}}=$ $\varepsilon^{k} \operatorname{vol}_{(1)}$, where $\operatorname{vol}_{(1)}=\operatorname{vol}_{\|\cdot\|_{(1)}}$. This and the monotonicity of the volume with respect to the norm imply that $\operatorname{vol}_{\|\cdot\|_{i}} \leq \varepsilon_{i}^{k} \operatorname{vol}_{(1)}$. Therefore, $\operatorname{vol}_{\|\cdot\|_{i}} \rightarrow 0=\operatorname{vol}_{\|\cdot\| \cdot}$.

Definition 4.11. By a density on an $n$-dimensional smooth manifold $M$ we mean a nonnegative measurable function $\nu: \Lambda^{n} T M \rightarrow \mathbb{R}$ whose restriction to each fiber is a seminorm, i.e., is symmetric and positive definite. $\left(\Lambda^{n} T M\right.$ is the one-dimensional vector bundle over $M$ whose fiber over a point $x \in M$ is the $n$th exterior power $\Lambda^{n} T_{x} M$.)

Such a structure can be integrated over a manifold in the same way as a differential form, and furthermore (unlike the case of a differential form), the integral does not depend on the manifold's orientation. Thus, the function $\nu$ determines a measure $\mu$ on $M$. We indicate this relationship between $\mu$ and $\nu$ by the formula $\mu=\int \nu$.

Definition 4.12. Let an $n$-dimensional Banach volume functional $\|\cdot\| \mapsto \operatorname{vol}_{\|\cdot\|}$ be fixed. Let $d$ be a Lipschitz metric on an $n$-dimensional smooth manifold $M$, and let $\varphi=\varphi_{d}$ be its tangent Finsler structure. Consider a density $\nu=\nu_{d}$ on $M$ whose value at an $n$-vector $\sigma \in \Lambda^{n} T_{x} M$ equals $\operatorname{vol}_{\left.\varphi\right|_{T_{x} M}}(\sigma)$ if $\left.\varphi\right|_{T_{x} M}$ is a seminorm, and 0 otherwise. We refer to the measure $\operatorname{vol}_{d}=\int \nu_{d}$ as the Finsler volume of $d$. 
Proposition 4.13. The measure $\operatorname{vol}_{d}$ described in Definition 4.12 is well defined and has the following properties.

1. Homogeneity: $\operatorname{vol}_{\lambda d}=\lambda^{n} \operatorname{vol}_{d}$ for every Lipschitz metric $d$ and every $\lambda>0$.

2. If $(M, d)$ and $\left(M^{\prime}, d^{\prime}\right)$ are manifolds with Lipschitz metrics and $f:(M, d) \rightarrow$ $\left(M^{\prime}, d^{\prime}\right)$ is a nonexpanding map, then $\operatorname{vol}_{d^{\prime}}(f(A)) \leq \operatorname{vol}_{d}(A)$ for every measurable $A \subset M$.

In particular, if $M^{\prime}=M$ and $d^{\prime} \leq d$, then $\operatorname{vol}_{d^{\prime}} \leq \operatorname{vol}_{d}$.

3. If $\left\{d_{i}\right\}$ is a monotone nondecreasing sequence of metrics on $M$ converging pointwise to a Lipschitz metric $d$, then $\operatorname{vol}_{d_{i}}(A) \rightarrow \operatorname{vol}(A)$ for every measurable $A \subset M$.

Proof. In order to verify that the definition is consistent, we need to prove that the density $\nu_{d}$ in Definition 4.12 is measurable. This follows from the fact that the function $\varphi_{d}: T M \rightarrow \mathbb{R}$ is measurable and the volume depends continuously on the seminorm (see Lemma $4.10(2))$.

Homogeneity follows from the corresponding property of a Banach volume (see Lemma $4.10(1))$.

To prove the second assertion, it suffices to verify that, at every point $x \in M$ where the map $f$ is differentiable, its Jacobian does not exceed 1 . Here by the Jacobian we mean the ratio of densities

$$
\frac{\left(d_{x} f\right)_{*} \nu_{d}(x)}{\nu_{d^{\prime}}(f(x))}
$$

This follows from property (4.7) of the Banach volume.

The third assertion follows from the fact that the tangent Finsler structures converge almost everywhere (see Proposition 2.91) and the volume depends continuously on the seminorm (see Lemma 4.10(2)).

Corollary 4.14. Definition 4.12 restricted to the class of Finsler manifolds defines a Finsler volume functional in the sense of Definition 4.1, Conversely, every Finsler volume functional can be obtained in this way.

Proof. The first statement follows from Proposition 4.13(2). The converse one follows from Proposition 4.6 .

Examples. Let $\Phi$ be a Finsler metric in a domain $U \subset \mathbb{R}^{n}$. Identifying $T_{x} U \simeq \mathbb{R}^{n}$ for all $x \in U$, we may regard $\Phi$ as a family of norms $\left\{\Phi_{x}\right\}_{x \in U}$ on $\mathbb{R}^{n}$. Denote by $B_{x}$ the unit ball of $\Phi_{x}$. Every Finsler volume has a continuous density $\rho$ with respect to the Lebesgue measure $m_{n}$, and it is related to the above coordinate-free density by the formula

$$
\rho(x)=\nu_{\Phi_{x}}\left(e_{1} \wedge e_{2} \wedge \cdots \wedge e_{n}\right),
$$

where $\left(e_{1}, e_{2}, \ldots, e_{n}\right)$ is the standard basis of $\mathbb{R}^{n}$.

In the case of the Busemann volume, the density equals

$$
\rho(x)=\frac{\omega_{n}}{m_{n}\left(B_{x}\right)},
$$

because the volume of the norm's unit ball equals $\omega_{n}$.

For the Holmes-Thompson volume, the density equals

$$
\rho(x)=\frac{m_{n}\left(B_{x}^{\circ}\right)}{\omega_{n}},
$$

where $B_{x}^{\circ}$ is the polar set of $B_{x}$. This follows from the definition of the Holmes-Thompson volume and the fact that $B_{x}^{\circ}$ is the unit ball of the dual norm $\|\cdot\|_{x}^{*}$ (modulo the standard identification $\left.\left(\mathbb{R}^{n}\right)^{*} \simeq \mathbb{R}^{n}\right)$. 
For the inscribed Riemannian volume, the density equals

$$
\rho(x)=\frac{\omega_{n}}{m_{n}\left(E_{x}\right)},
$$

where $E_{x}$ is the John ellipsoid of the norm $\Phi_{x}$.

\section{$\S 5$. Filling VOLUMES}

Let an $n$-dimensional Finsler volume functional be fixed. Then, by Definition 4.12, for every $n$-dimensional manifold $M$ with a Lipschitz metric $d$ there is an associated measure $\operatorname{vol}_{d}$ on $M$.

Definition 5.1. Let $\mathfrak{M}$ be a class of compact $n$-dimensional manifolds with Lipschitz metrics. Let $S$ be an $(n-1)$-dimensional manifold and $d_{0}$ a Lipschitz metric on $S$. By the filling volume of $d_{0}$ within the class $\mathfrak{M}$ (with respect to the given volume functional) we mean the quantity

$$
\inf \left\{\operatorname{vol}_{d}(M):(M, d) \in \mathfrak{M}, \partial M=S,\left.d\right|_{S \times S} \geq d_{0}\right\} .
$$

We say that a manifold $(M, d) \in \mathfrak{M}$ is a minimal filling within $\mathfrak{M}$ if $\operatorname{vol}_{d}(M)$ equals the filling volume of the metric $\left.d\right|_{\partial M \times \partial M}$ within $\mathfrak{M}$.

The definition of the filling volume given in the Introduction corresponds to the filling volume within the class of Riemannian manifolds.

Theorem 5.2. Let $M$ be an $n$-dimensional manifold, let $S=\partial M$, and let $d_{0}$ be a Lipschitz metric on $S$. Then:

1. the filling volume of $d_{0}$ within the class of all Lipschitz metrics on $M$ equals its filling volume within the class of smooth strictly convex Finsler metrics;

2. if the volume functional is the inscribed Riemannian volume, then the filling volume of $d_{0}$ within the class of all Lipschitz metrics on $M$ equals its filling volume within the class of Riemannian metrics.

Proof. 1. Let $d$ be a Lipschitz metric on $M$ such that $\left.d\right|_{S \times S} \geq d_{0}$. It suffices to prove that, for every $\varepsilon>0$, there is a smooth strictly convex Finsler metric $\tilde{d}$ on $M$ such that $\left.\tilde{d}\right|_{S \times S} \geq\left. d\right|_{S \times S}$ and $\operatorname{vol}_{\tilde{d}}(M) \leq \operatorname{vol}_{d}(M)+\varepsilon$. We may assume that $d$ is a length metric, because replacing a metric by the induced length does not decrease the distances and preserves the volume (by Proposition 2.12).

We attach to $M$ a "collar" $M^{+}=S \times[0,1]$ by identifying the sets $S \subset M$ and $S \times\{0\} \subset S \times[0,1]$, and we construct a smooth Riemannian metric $d^{+}$on $M^{+}$such that

(1) $\operatorname{vol}_{d^{+}}\left(M^{+}\right)<\varepsilon$

(2) $d^{+}\left((x, t)\left(x^{\prime}, t^{\prime}\right)\right)>d\left(x, x^{\prime}\right)$ for all $x, x^{\prime} \in S, t, t^{\prime} \in[0,1]$ with $(x, t) \neq\left(x^{\prime}, t^{\prime}\right)$.

For instance, $d^{+}$can be defined as the product metric of $\left(S, d_{0}^{+}\right) \times[0, \theta]$, where $d_{0}^{+}$is a Riemannian metric on $S, d_{0}^{+} \geq\left. 2 d\right|_{S \times S}, 0<\theta<\varepsilon / \operatorname{vol}\left(S, d_{0}^{+}\right)$.

The enlarged manifold $M^{\prime}=M \cup M^{+}$is equipped with a metric $d^{\prime}$ obtained by gluing the metrics $d$ on $M$ and $d^{+}$on $M^{+}$. The gluing procedure is described in [11, §3.1]; here we use only the fact that the result is a length metric which is isometric to the initial metrics in the interiors of the two manifolds. Observe that

$$
d^{\prime}\left((x, t),\left(x^{\prime}, t^{\prime}\right)\right) \geq d(x, y)
$$

for all $x, x^{\prime} \in S, t, t^{\prime} \in[0,1]$, since this inequality holds for both metrics before gluing.

Let $\delta>0$ be such that $d^{+}\left((x, 1),\left(x^{\prime}, \frac{1}{2}\right)\right) \geq d\left(x, x^{\prime}\right)+\delta$ for all $x, x^{\prime} \in S$. For the metric $d^{\prime}$, we construct a sequence $\left\{\varphi_{i}\right\}$ of smooth Finsler structures as in Proposition 2.14; in addition, we choose $\varphi_{i}$ so that they agree with the Riemannian structure of $d^{+}$on the set $S \times\left[\frac{1}{2}, 1\right]$ (see Remark 2.15). Let $d_{i}=d_{\varphi_{i}}$. By properties 1 and 3 in Proposition 2.14, the densities of the Finsler volumes determined by the metrics $d_{i}$ are uniformly bounded and 
converge pointwise to the density of $\operatorname{vol}_{d^{\prime}}$. Therefore, $\operatorname{vol}_{d_{i}}\left(M^{\prime}\right) \rightarrow \operatorname{vol}_{d^{\prime}}\left(M^{\prime}\right)$. Hence, for all sufficiently large $i$ we have $\operatorname{vol}_{d_{i}}\left(M^{\prime}\right)<\operatorname{vol}_{d}(M)+\varepsilon$.

For all sufficiently large $i$ we have $d_{i} \geq d^{\prime}-\delta$ everywhere on $M^{\prime}$ (see property 2 in Proposition 2.14). It follows that $d_{i}((x, 1),(y, 1)) \geq d(x, y)$ for all $x, y \in S$. Indeed, let $\gamma$ be a shortest curve of the metric $d_{i}$ connecting $(x, 1)$ and $(y, 1)$. If this curve lies entirely in the set $S \times\left[\frac{1}{2}, 1\right]$, then its length is at least $d^{+}((x, 1),(y, 1)) \geq d(x, y)$. Otherwise, let $\left(x^{\prime}, \frac{1}{2}\right)$ and $\left(y^{\prime}, \frac{1}{2}\right)$ be the points of the intersection of $\gamma$ with the set $S \times\left\{\frac{1}{2}\right\}$ nearest to $x$ and $y$, respectively. Then

$$
\begin{aligned}
d_{i}((x, 1),(y, 1)) & =d^{+}\left((x, 1),\left(x^{\prime}, \frac{1}{2}\right)\right)+d_{i}\left(\left(x^{\prime}, \frac{1}{2}\right),\left(y^{\prime}, \frac{1}{2}\right)\right)+d^{+}\left((y, 1),\left(y^{\prime}, \frac{1}{2}\right)\right) \\
& \geq\left(d\left(x, x^{\prime}\right)+\delta\right)+\left(d^{\prime}\left(\left(x^{\prime}, \frac{1}{2}\right),\left(y^{\prime}, \frac{1}{2}\right)\right)-\delta\right)+\left(d\left(y, y^{\prime}\right)+\delta\right) \\
& \geq\left(d\left(x, x^{\prime}\right)+\delta\right)+\left(d\left(x^{\prime}, y^{\prime}\right)-\delta\right)+\left(d\left(y, y^{\prime}\right)+\delta\right)>d(x, y) .
\end{aligned}
$$

Here the second inequality follows from (5.3) and the final one from the triangle inequality for $d$.

Let $f: M \rightarrow M^{\prime}$ be a diffeomorphism mapping $S \subset M$ to $S \times\{1\} \subset M^{\prime}$ in the natural way. Let $\tilde{d}_{i}=f^{*} d_{i}$ be the metric on $M$ corresponding to $d_{i}$ under this diffeomorphism. Then for a sufficiently large $i$ the metric $\tilde{d}=\tilde{d}_{i}$ satisfies the conditions $\left.\tilde{d}\right|_{S \times S} \geq\left. d\right|_{S \times S}$ and $\operatorname{vol}_{\tilde{d}}(M)<\operatorname{vol}_{d}(M)+\varepsilon$, hence the result.

2. For each point $x \in M$, consider the norm on $T_{x} M$ defined as the restriction of the Finsler structure of the metric $\tilde{d}$ constructed above. Let $E_{x}$ be the John ellipsoid of this norm (see Example 4.4). Let $d_{r}$ be a Riemannian metric on $M$ generated by the family of ellipsoids $\left\{E_{x}\right\}_{x \in M}$. Then $d_{r} \geq \tilde{d}$, whence $\left.d_{r}\right|_{S \times S} \geq\left. d\right|_{S \times S}$. Furthermore, since the chosen Finsler volume is the inscribed Riemannian volume, we have $\operatorname{vol}_{d_{r}}(M)=\operatorname{vol}_{\tilde{d}}(M)<\operatorname{vol}_{d}(M)+\varepsilon$. By the uniqueness of the John ellipsoid, the Riemannian structure of $d_{r}$ is continuous; it can be smoothed so that the inequalities $\left.d_{r}\right|_{S \times S} \geq\left. d\right|_{S \times S}$ and $\operatorname{vol}_{d_{r}}(M)<\operatorname{vol}_{d}(M)+\varepsilon$ survive. Thus, the filling volume is realized by smooth Riemannian metrics, hence the result.

Definition 5.4. Let $M$ be a smooth manifold, $X$ a Banach space, $f: M \rightarrow X$ a Lipschitz map. Let a Finsler volume functional be fixed. By the area of a map (or surface) $f$ we mean the quantity area $(f)=\operatorname{vol}_{d}(M)$, where $d$ is the metric on $M$ given by $d(x, y)=\|f(x)-f(y)\|$, and $\mathrm{vol}_{d}$ is the measure determined by this Lipschitz metric and the chosen Finsler volume functional (see Definition 4.12).

If the Banach space $X$ is dual to a separable one (for example, has the form $L^{\infty}(\mu)$, where $\mu$ is a finite measure), then the area of a map can be expressed in terms of its weak differential in the standard way. Namely, the following statement is true.

Proposition 5.5. Let $X$ be a Banach space that is dual to a separable one, $M$ a smooth manifold, $f: M \rightarrow X$ a Lipschitz map. Then area $(f)=\int_{M} \nu_{f}$, where $\nu_{f}$ is a density on $M$ whose value at an $n$-vector $\sigma \in T_{x} M$ equals

$$
\nu_{f}(\sigma)=\operatorname{vol}_{\left(d_{x}^{w} f\right)^{*}\|\cdot\|_{X}}
$$

provided $f$ is weakly differentiable at $x$. Here the star denotes the pull-back of a seminorm by a linear map; that is, $\left(d_{x}^{w} f\right)^{*}\|\cdot\|_{X}$ is the seminorm $\|\cdot\|$ on $T_{x} M$ given by $\|v\|=$ $\left\|d_{x}^{w} f(v)\right\|_{X}$.

Proof. This follows from the definition of area and Theorem 3.7

Theorem 5.6. For every Finsler volume functional, the following holds true. Let $X=$ $L^{\infty}(\mu)$, where $\mu$ is a finite measure on an arbitrary set, $M$ is a smooth manifold, and $S=\partial M$. 
1. Let $d_{0}$ be a Lipschitz metric on $S$ and $f:\left(S, d_{0}\right) \rightarrow X$ an isometric map. Then the filling volume of $\left(S, d_{0}\right)$ within the class of Lipschitz (or, equivalently, smooth strictly convex Finsler) metrics on $M$ equals

$$
\inf \left\{\operatorname{area}(F): F: M \rightarrow X \text { is Lipshitz, }\left.F\right|_{S}=f\right\} .
$$

2. Let $d$ be a Lipschitz metric on $M$. Then for every isometric map $F:(M, d) \rightarrow X$, the following holds: $(M, d)$ is a minimal filling within the class of all manifolds with Lipschitz metrics if and only if $F$ realizes a minimum of the area among all Lipschitz surfaces in $X$ having the same boundary.

Proof. 1. Let $F: M \rightarrow X$ be a Lipschitz map, and let $\left.F\right|_{S}=f$. Consider a Lipschitz metric $d$ on $M$ induced by $F$ (i.e., given by $d(x, y)=\|F(x)-F(y)\|)$. By the definition of area, $\operatorname{area}(F)=\operatorname{vol}(M, d)$. Since the map $f=\left.F\right|_{S}$ is isometric with respect to $d_{0}$, we have $\left.d\right|_{S \times S}=d_{0}$. Therefore, the filling volume of $\left(S, d_{0}\right)$ does not exceed area $(F)$. Since $F$ is arbitrary, the filling volume does not exceed the infimum of such areas.

Conversely, if $d$ is a Lipschitz metric on $M$ such that $\left.d\right|_{S \times S} \geq d_{0}$, then, by Proposition 1.6. there exists a nonexpanding map $F:(M, d) \rightarrow X$. Since $F$ is nonexpanding, the metric on $M$ induced by it does not exceed $d$; therefore, area $(F) \leq \operatorname{vol}(M, d)$. Taking the infimum over $(M, d)$ yields the desired result.

2. This follows trivially from the first assertion of the theorem.

With the inscribed Riemannian volume used as the definition of volume, Theorem 5.6 and the second assertion of Theorem 5.2 imply the following.

Corollary 5.7. Let $X=L^{\infty}(\mu)$, where $\mu$ is a finite measure on an arbitrary set, $M$ is a smooth manifold, and $S=\partial M$. Define the area of a surface in $X$ as the area determined by the inscribed Riemannian volume functional.

1. Let $d_{0}$ be a Lipschitz metric on $S$, and let $f:\left(S, d_{0}\right) \rightarrow X$ be an isometric map. Then the filling volume of $\left(S, d_{0}\right)$ within the class of Riemannian metrics on $M$ equals

$$
\inf \left\{\operatorname{area}(F): F: M \rightarrow X \text { is Lipschitz, }\left.F\right|_{S}=f\right\} .
$$

2. Let $d$ be a Riemannian metric on $M$. Then for every isometric map $F:(M, d) \rightarrow$ $X$, the following holds: $(M, d)$ is a minimal filling within the class of all Riemannian manifolds if and only if $F$ realizes the infimum of the area among all Lipschitz surfaces in $X$ having the same boundary.

\section{$\S 6$. Semiellipticity}

Definition 6.1. We say that an $n$-dimensional Finsler volume functional is semielliptic over $\mathbf{Z}$ if the associated $n$-dimensional area functional in every finite-dimensional Banach space $V$ satisfies the following: the image $L\left(D^{n}\right)$ of the standard disk $D^{n} \subset \mathbb{R}^{n}$ under any injective linear map $L: \mathbb{R}^{n} \rightarrow V$ minimizes the area among all oriented Lipschitz surfaces in $V$ having the same boundary.

We say that an $n$-dimensional Finsler volume functional is topologically semielliptic if a similar minimality occurs in the class of all Lipschitz surfaces parametrized by the disk $D^{n}$, and it is semielliptic over $\mathbb{R}$ if it occurs in the class of all Lipschitz chains with real coefficients.

In other words, a volume functional is semielliptic if the area integrand determined by it is semielliptic in any finite-dimensional Banach space. It is plausible that in this case the area integrand is elliptic in spaces with strictly convex norms. The ellipticity of area integrands plays an important role in the theory of minimal surfaces (see $[1 ; 16$, Chapter 5]). In [8], relationships between semiellipticity and filling volumes, as well as asymptotic volumes of periodic metrics, were shown. 
Obviously, semiellipticity over $\mathbb{R}$ implies that over $\mathbb{Z}$. The converse is not true; a counterexample was constructed in $[9$. Semiellipticity over $\mathbb{Z}$ obviously implies topological semiellipticity, and for all $n \geq 3$, these two properties are equivalent [17, Appendix 2, Proposition $\mathrm{A}^{\prime}$ ].

The semiellipticity of the Busemann and Holmes-Thompson volumes is a widely open question. In codimension 1, both volumes are semielliptic (this is equivalent to the convexity of section bodies and projection bodies, respectively; see [13, 23]). The twodimensional Holmes-Thompson volume is topologically semielliptic but is not semielliptic over $\mathbb{R}[8$. The question as to whether the Busemann volume is semielliptic in codimensions greater than 1 is completely open by now.

Our purpose in this section is to prove the following theorem.

Theorem 6.2. Let $X$ be a normed vector space and $Y \subset X$ an n-dimensional linear subspace. Then there exists a linear map $P: X \rightarrow Y$ such that the following is true.

1. $P$ is a projector onto $Y$, that is, $\left.P\right|_{Y}=i d_{Y}$.

2. For every Lipshitz map $f: M \rightarrow X$, where $M$ is a smooth $n$-dimensional manifold, we have area $(P \circ f) \leq$ area $(f)$, where area is the area defined by the inscribed Riemannian volume functional.

Consequently, the inscribed Riemannian volume functional is semielliptic over $\mathbb{R}$.

Proof. The proof is based on ideas from [7]. First, assume that the space $X$ is dual to a separable one. Then, by Proposition 5.5, it suffices to construct a projector that does not increase the $n$-dimensional area on $n$-dimensional linear subspaces of $X$. We need the following lemma.

Lemma 6.3 (see [7, Lemma 1.3]). Let $(V,\|\cdot\|)$ be an $n$-dimensional normed vector space. Then there exists a finite collection $\left\{L_{i}\right\}_{i=1}^{N}$ of nonexpanding linear functions $L_{i}:(V,\|\cdot\|) \rightarrow \mathbb{R}$ and a collection $\left\{\lambda_{i}\right\}_{i=1}^{N}$ of positive numbers such that $\sum \lambda_{i}=n$ and $\sum \lambda_{i} L_{i}^{2}(v) \geq\|v\|$ for all $v \in V$.

Applying this lemma to a subspace $Y$ equipped with the restriction of the norm $\|\cdot\|_{X}$, we get a collection of nonexpanding linear maps $L_{i}: Y \rightarrow \mathbb{R}$ and coefficients $\lambda_{i} \geq 0$, $i=1, \ldots, N$, such that $\sum \lambda_{i}=n$ and $\sum \lambda_{i} L_{i}^{2} \geq\|\cdot\|_{X}^{2}$ everywhere on $Y$. By the HahnBanach theorem, the functions $L_{i}$ have nonexpanding linear extensions $\tilde{L}_{i}: X \rightarrow \mathbb{R}$. Consider the quadratic form $Q$ on $X$ given by $Q(x)=\sum \lambda_{i} \tilde{L}_{i}^{2}$. We denote by area ${ }_{Q}$ the $n$-dimensional area with respect to the Euclidean seminorm $\sqrt{Q}$.

We prove that area $_{Q} \leq$ area on every $n$-dimensional linear subspace $W \subset X$. Recall that here by area we mean the inscribed Riemannian volume of the induced metric. Let $|\cdot|_{W}$ be the Euclidean norm on $W$ whose unit ball is the John ellipsoid of the restriction of the norm $\|\cdot\|_{X}$ to $W$. Then on $W$ we have $|\cdot|_{W} \geq\|\cdot\|_{X}$, and $\operatorname{vol}_{|\cdot|_{W}}=\operatorname{vol}_{\|\cdot\|_{X}}$ by definition. The inequality $|\cdot|_{W} \geq\|\cdot\|_{X}$ implies that the restrictions $\left.\tilde{L}_{i}\right|_{W}$ are nonexpanding with respect to $|\cdot|_{W}$; therefore,

$$
\operatorname{trace}_{||_{W}}\left(\left.L_{i}^{2}\right|_{W}\right) \leq 1
$$

whence

$$
\operatorname{trace}_{|\cdot|_{W}}\left(\left.Q\right|_{W}\right) \leq \sum \lambda_{i}=n .
$$

By the Cauchy inequality, this implies that

$$
\operatorname{det}_{|\cdot|_{W}}\left(\left.Q\right|_{W}\right) \leq\left(\frac{1}{n} \operatorname{trace}_{|\cdot|_{W}}\left(\left.Q\right|_{W}\right)\right)^{n} \leq 1 .
$$

(Here trace $\left.\right|_{||_{W}}$ and $\operatorname{det}_{\left.\left.\right|_{\mid}\right|_{W}}$ denote (respectively) the trace and the determinant of a quadratic form in the Euclidean space $(W,|\cdot| W)$.) Therefore, the volume form on $W$ determined by the quadratic form $\left.Q\right|_{W}$ does not exceed the volume form determined by the norm $|\cdot|_{W}$. Thus, area $_{Q} \leq$ area on $W$. 
The inequality $Q(x) \geq\|x\|_{X}^{2}$ for all $x \in Y$ implies that, on the subspace $Y$, we have area $_{Q} \geq$ area, whence area $Q=$ area on $Y$.

Let $P: X \rightarrow Y$ be the orthogonal projection of $X$ onto $Y$ with respect to the quadratic form $Q$. Since an orthogonal projection does not increase the lengths of the vectors, it does not increase the area with respect to $Q$. Hence,

$$
\operatorname{area}(P(A))=\operatorname{area}_{Q}(P(A)) \leq \operatorname{area}_{Q}(A) \leq \operatorname{area}(A)
$$

for every measurable set $A$ contained in an $n$-dimensional linear subspace $W \subset X$. Therefore, the map $P$ is a desired one. Thus, we have proved the theorem in the case where the space $X$ is dual to a separable one.

Now we consider the general case. Let $S=\left\{s_{i}\right\}_{i=1}^{\infty}$ be a countable dense subset of the unit sphere of $Y$. For each $i$, by the Hahn-Banach theorem there exists a nonexpanding linear map $f_{i}: X \rightarrow \mathbb{R}$ such that $f_{i}\left(s_{i}\right)=1$. We define a linear map $f: X \rightarrow \ell_{\infty}$ by

$$
f(x)=\left(f_{1}(x), f_{2}(x), \ldots\right) .
$$

This map is nonexpanding because so are all the maps $f_{i}$. Furthermore, the restriction $\left.f\right|_{Y}$ is isometric. Indeed, for every $x \in Y$ such that $\|x\|_{X}=1$ we have

$$
\|f(x)\|_{\infty}=\sup _{i} f_{i}(x) \geq \sup _{i}\left(f_{i}\left(s_{i}\right)-\left\|x-s_{i}\right\|_{X}\right)=\sup _{i}\left(1-\left\|x-s_{i}\right\|_{X}\right)=1,
$$

because $f$ is nonexpanding and $S$ is dense in the unit sphere of $Y$. Since the space $\ell_{\infty}$ is dual to a separable one, the case already proved shows that there exists a projector $P_{0}: \ell_{\infty} \rightarrow f(Y)$ that does not increase $n$-dimensional areas. Then the map $P=$ $\left(\left.f\right|_{Y}\right)^{-1} \circ P_{0} \circ f$ is a desired one.

\section{REFERENCES}

[1] F. J. Almgren, Jr., Existence and regularity almost everywhere of solutions to elliptic variational problems among surfaces of varying topological type and singularity structure, Ann. of Math. (2) 87 (1968), 321-391. MR0225243 (37:837)

[2] J. C. Álvarez-Paiva and G. Berck, What is wrong with the Hausdorff measure in Finsler spaces, Preprint, 2004, arXiv:math.DG/0408413.

[3] J. C. Álvarez-Paiva and A. C. Thompson, Volumes on normed and Finsler spaces, A Sampler of Riemann-Finsler Geometry (D. Bao, R. Bryant, S.-S. Chern, and Z. Shen, eds.), Math. Sci. Res. Inst. Publ., No. 50, Cambridge Univ. Press, Cambridge, 2004, pp. 1-48. MR2132656 (2006c:53079)

[4] Y. Benyamini and J. Lindenstrauss, Geometric nonlinear functional analysis. Vol. 1, Amer. Math. Soc. Colloq. Publ., vol. 48, Amer. Math. Soc., Providence, RI, 2000. MR1727673 (2001b:46001)

[5] A. Besicovitch, On two problems of Loewner, J. London Math. Soc. 27 (1952), 141-144. MR0047126 $(13: 831 d)$

[6] D. Burago and S. Ivanov, Isometric embeddings of Finsler manifolds, Algebra i Analiz 5 (1993), no. 1, 179-192; English transl., St. Petersburg Math. J. 5 (1994), no. 1, 159-169. MR1220494 (94e:53073)

[7] $\longrightarrow$ On asymptotic volume of tori, Geom. Funct. Anal. 5 (1995), 800-808. MR1354290 (96h:53041)

[8] On asymptotic volume of Finsler tori, minimal surfaces in normed spaces, and symplectic filling volume, Ann. of Math. (2) 156 (2002), 891-914. MR.1954238 (2003k:53088)

[9] Gaussian images of surfaces and ellipticity of surface area functionals, Geom. Funct. Anal. 14 (2004), 469-490. MR2100668 (2006b:53099)

[10] _ Boundary rigidity and filling volume minimality of metrics close to a flat one (to appear).

[11] D. Yu. Burago, Yu. D. Burago, and S. V. Ivanov, A course in metric geometry, Inst. Komp'yut. Issled., Moscow-Izhevsk, 2004; English transl., Grad. Stud. in Math., vol. 33, Amer. Math. Soc., Providence, RI, 2001. MR 1835418 (2002e:53053)

[12] H. Busemann, Intrinsic area, Ann. of Math. (2) 48 (1947), 234-267. MR0020626 (8:573a)

[13] _ A theorem on convex bodies of the Brunn-Minkowski type, Proc. Nat. Acad. Sci. U.S.A. 35 (1949), 27-31. MR0028046 (10:395c)

[14] H. Busemann, G. Ewald, and G. C. Shephard, Convex bodies and convexity on Grassmann cones. I-IV, Math. Ann. 151 (1963), 1-41. MR0157286 (28:522a) 
[15] G. De Cecco and G. Palmieri, LIP manifolds: From metric to Finslerian structure, Math. Z. 218 (1995), 223-237. MR1318157 (96a:53090)

[16] H. Federer, Geometric measure theory, Grundlehren Math. Wiss., Bd. 153, Springer-Verlag New York, Inc., New York, 1969. MR0257325 (41:1976)

[17] M. Gromov, Filling Riemannian manifolds, J. Differential Geom. 18 (1983), 1-147. MR0697984 (85h:53029)

[18] R. D. Holmes and A. C. Thompson, n-dimensional area and content in Minkowski spaces, Pacific J. Math. 85 (1979), 77-110. MR0571628 (81k:52023)

[19] S. Ivanov, On two-dimensional minimal fillings, Algebra i Analiz 13 (2001), no. 1, 26-38; English transl., St. Petersburg Math. J. 13 (2002), no. 1, 17-25. MR.1819361 (2002b:58016)

[20] F. John, Extremum problems with inequalities as subsidiary conditions, Studies and Essays Presented to R. Courant on his 60th Birthday, January 8, 1948, Intersci. Publ., Inc., New York, NY, 1948, pp. 187-204. MR0030135(10:719b)

[21] R. Michel, Sur la rigidité imposée par la longuer des géodésiques, Invent. Math. 65 (1981/82), 71-83. MR0636880 (83d:58021)

[22] P. Pu, Some inequalities in certain nonorientable Riemannian manifolds, Pacific J. Math. 2 (1952), 55-71. MR0048886 (14:87e)

[23] A. C. Thompson, Minkowski geometry, Encyclopedia Math. Appl., vol. 63, Cambridge Univ. Press, Cambridge, 1996. MR1406315 (97f:52001)

[24] R. Schneider, On the Busemann area in Minkowski spaces, Beiträge Algebra Geom. 42 (2001), 263-273. MR.1824764 (2002k:52006)

St. Petersburg Branch, Steklov Mathematical Institute, Russian Academy of Sciences, Fontanka 27, St. Petersburg 191023, Russia

E-mail address: svivanov@pdmi.ras.ru

Received 29/MAY/2007

Translated by THE AUTHOR 